\title{
PIV Measurements in the Atmospheric Boundary Layer within and above a Mature Corn Canopy. Part I: Statistics and Energy Flux
}

\author{
R. van Hout,* W. Zhu, L. LuZnik, AND J. Katz \\ Center for Environmental and Applied Fluid Mechanics, Department of Mechanical Engineering, The Johns Hopkins University, \\ Baltimore, Maryland \\ J. KLEISSL ${ }^{+}$AND M. B. PARlangE $\#$ \\ Center for Environmental and Applied Fluid Mechanics, Department of Geography and Environmental Engineering, The Johns \\ Hopkins University, Baltimore, Maryland
}

(Manuscript received 13 May 2005, in final form 21 November 2006)

\begin{abstract}
Particle image velocimetry (PIV) measurements just within and above a mature corn canopy have been performed to clarify the small-scale spatial structure of the turbulence. The smallest resolved scales are about 15 times the Kolmogorov length scale $(\eta \approx 0.4 \mathrm{~mm})$, the Taylor microscales are about $100 \eta$, and the Taylor scale Reynolds numbers range between $R_{\lambda}=2000$ and 3000. The vertical profiles of mean flow and turbulence parameters match those found in previous studies. Frequency spectra, obtained using the data as time series, are combined with instantaneous spatial spectra to resolve more than five orders of magnitude of length scales. They display an inertial range spanning three decades. However, the small-scale turbulence in the dissipation range exhibits anisotropy at all measurement heights, in spite of apparent agreement with conditions for reaching local isotropy, following a high-Reynolds-number wind tunnel study. Directly calculated subgrid-scale (SGS) energy flux, determined by spatially filtering the PIV data, increases significantly with decreasing filter size, providing support for the existence of a spectral shortcut that bypasses the cascading process and injects energy directly into small scales. The highest measured SGS flux is about $40 \%$ of the estimated energy cascading rate as determined from a $-5 / 3$ fit to the spectra. Terms appearing in the turbulent kinetic energy budget that can be calculated from the PIV data are in agreement with previous results. Evidence of a very strong correlation between dissipation rate and out-of-plane component of the vorticity is demonstrated by a striking similarity between their time series.
\end{abstract}

\section{Introduction}

Turbulent boundary layer flow measurements inside and above canopies have been performed extensively in the last three decades as summarized in Raupach and Thom (1981) and Finnigan (2000). Several studies have

\footnotetext{
* Current affiliation: Faculty of Mechanical Engineering, Technion-IIT, Technion City, Haifa, Israel.

+ Current affiliation: Department of Mechanical and Aerospace Engineering, University of California, San Diego, La Jolla, California.

\# Current affiliation: School of Architecture, Civil and Environmental Engineering, Ecole Polytechnique Fédérale de Lausanne, Lausanne, Switzerland.
}

Corresponding author address: J. Katz, Department of Mechanical Engineering, The Johns Hopkins University, $3400 \mathrm{~N}$. Charles St., Baltimore, MD 21218.

E-mail:katz@poseidon.me.jhu.edu focused on corn canopies (e.g., Shaw et al. 1974a,b, 1983; Wilson et al. 1982). Besides fundamental insights on the structure of turbulent flow over rough boundaries in general, this knowledge is essential for predicting scalar exchange between the atmosphere and the canopy. Furthermore, turbulent entrainment and dispersal of pollen grains (anemophilous) into the atmosphere is significant for ecological studies on genetic diversity (Wolfenbarger and Phifer 2000) as well as its impact on allergies.

Past measurements of atmospheric turbulence in near-neutral flow within and above different plant canopies have revealed a striking consistency of the mean flow and turbulence characteristics (Raupach et al. 1996). They show that the vertical profiles of normalized mean streamwise velocity exhibit an inflection point at canopy height that generates flow instabilities. The actual profile depends on the canopy density, which is characterized by the leaf area index (LAI) and

DOI: $10.1175 / \mathrm{JAS} 3989.1$

(C) 2007 American Meteorological Society 
the related projected frontal area index (PFAI), PFAI $\approx \mathrm{LAI} / 2$ (Poggi et al. 2004). The roughness layer extends up to three canopy heights (Raupach et al. 1991) and includes a constant Reynolds stress layer of one to two canopy heights. Brunet et al. (1994) observed a similarity between the roughness layer turbulence statistics and those of a plane mixing layer, and consequently Raupach et al. (1996) proposed a canopy mixing layer analogy. Various subsequent experiments have strengthened this picture.

Characteristics of small-scale flow structures have been investigated in high-Reynolds-number turbulent flows. For example, local isotropy has been confirmed using spectral characteristics in a wind tunnel study of a high-Reynolds-number, rough-wall turbulent boundary flow by Saddoughi and Veeravalli (1994). For canopy flows, as discussed in detail by Finnigan (2000), the assumption of isotropy seems to be inherently violated within canopies. Here, besides the energy cascading down the inertial subrange, two additional processes cause direct transfer of kinetic energy from large scales to small scales, bypassing the cascading process. The first process is associated with interaction of mean flow with canopy elements, which converts some of the mean kinetic energy directly into finescale turbulence (wake production). The second process involves direct conversion of turbulent kinetic energy of large-scale eddies to finescale turbulence (spectral shortcut), also by the canopy elements. As a result of these two processes, the shape of the spectrum is altered and deviates in the inertial subrange from the classical $-5 / 3$ behavior (Finnigan 2000). In wind tunnel model canopies, this spectral shortcut has been shown to increase the energy level in the inertial and dissipation ranges of the spectrum (Raupach et al. 1986).

The small-scale spatial structure in canopy flows, especially in natural canopies, is still not well resolved (Finnigan 2000). Energy spectra have been obtained both in the field (e.g., Shaw et al. 1974a; Amiro 1990) and in wind tunnels (e.g., Brunet et al. 1994) using single point sensors. Based on the ratios of turbulent spectral density between the streamwise and wallnormal components in the inertial ( $-5 / 3$ slope) range, isotropy was not attained. However, the highReynolds-number field experiments did not resolve the small scales, while the Reynolds numbers in the wind tunnel studies were too low for the development of local isotropy. For example, the split hot-film anemometers used by Shaw et al. (1974a) and Wilson et al. (1982) had a physical dimension of $0.9 \mathrm{~cm}$ and a sampling frequency $f$ leading to $U / f \sim 0.2 \mathrm{~m}$, where $U$ is the mean velocity. Sonic anemometers that have typical pathlengths of $10-15 \mathrm{~cm}$ have been used for studying forest canopies (e.g., Collineau and Brunet 1993). Thus, the kinetic energy at the scale of canopy element wakes has not been resolved (Finnigan 2000).

In this study we use a time series of particle image velocimetry (PIV) measurements just within and above a mature corn canopy in order to resolve both the largeand small-scale turbulence. Two-dimensional PIV measures the instantaneous spatial distribution of two velocity components at a resolution that depends on several system parameters (details follow), $\sim 2.8 \mathrm{~mm}$ in the present study. The large-scale structures are resolved by invoking Taylor's hypothesis on the time series, whereas the small scales are resolved spatially. The data compare well with other sensors and previously observed trends. We use the energy spectra to estimate the energy flux cascading down in the inertial range, not to be mistaken as the total dissipation (Finnigan 2000). Associated small-scale turbulence parameters are determined using this dissipation rate. However, we demonstrate that local isotropy is not obtained at the smallest resolved scales, although several tests seem to suggest the opposite. Furthermore, spatial filtering of the instantaneous velocity distribution enables us to calculate the subgrid-scale (SGS) stresses and energy flux, that is, the energy transferred from resolved to subgrid scales. The SGS energy flux increases with decreasing filter size, reaching $40 \%$ of the spectral estimate of cascading energy flux, supporting the claim of bypass mechanisms. Finally, we use a sample time series of velocity fluctuations, Reynolds stresses, dissipation, and vorticity magnitude to demonstrate a weak correlation between dissipation and stress and a strong correlation between the dissipation rate and the out-ofplane component of the vorticity.

\section{Description of the field experiments}

\section{a. Setup and conditions}

The field measurements were performed from 10 July 2003 until 22 July 2003, on the eastern shore of the Chesapeake Bay, at a latitude of $38^{\circ} 35^{\prime} 31^{\prime \prime} \mathrm{N}$ and a longitude of $75^{\circ} 51^{\prime} 55^{\prime \prime} \mathrm{W}$. The site was a flat, irrigated, 1.2$\mathrm{km}^{2}$ circular field with a diameter of about $800 \mathrm{~m}$. Half of this field was planted with corn, and the other half with potatoes. During the course of the experiments the corn plants were fully matured and pollinating. The location of the various instruments relative to the center of the field is shown in Fig. 1. The meteorological station consisted of two 3D Campbell Scientific sonic anemometers/thermometers (CSAT), a Vaisala HMP45C hygrometer/thermometer, an RM Young 03105 propeller wind vane (RMY), and a Texas Electronics rain gauge. This station and the PIV system 


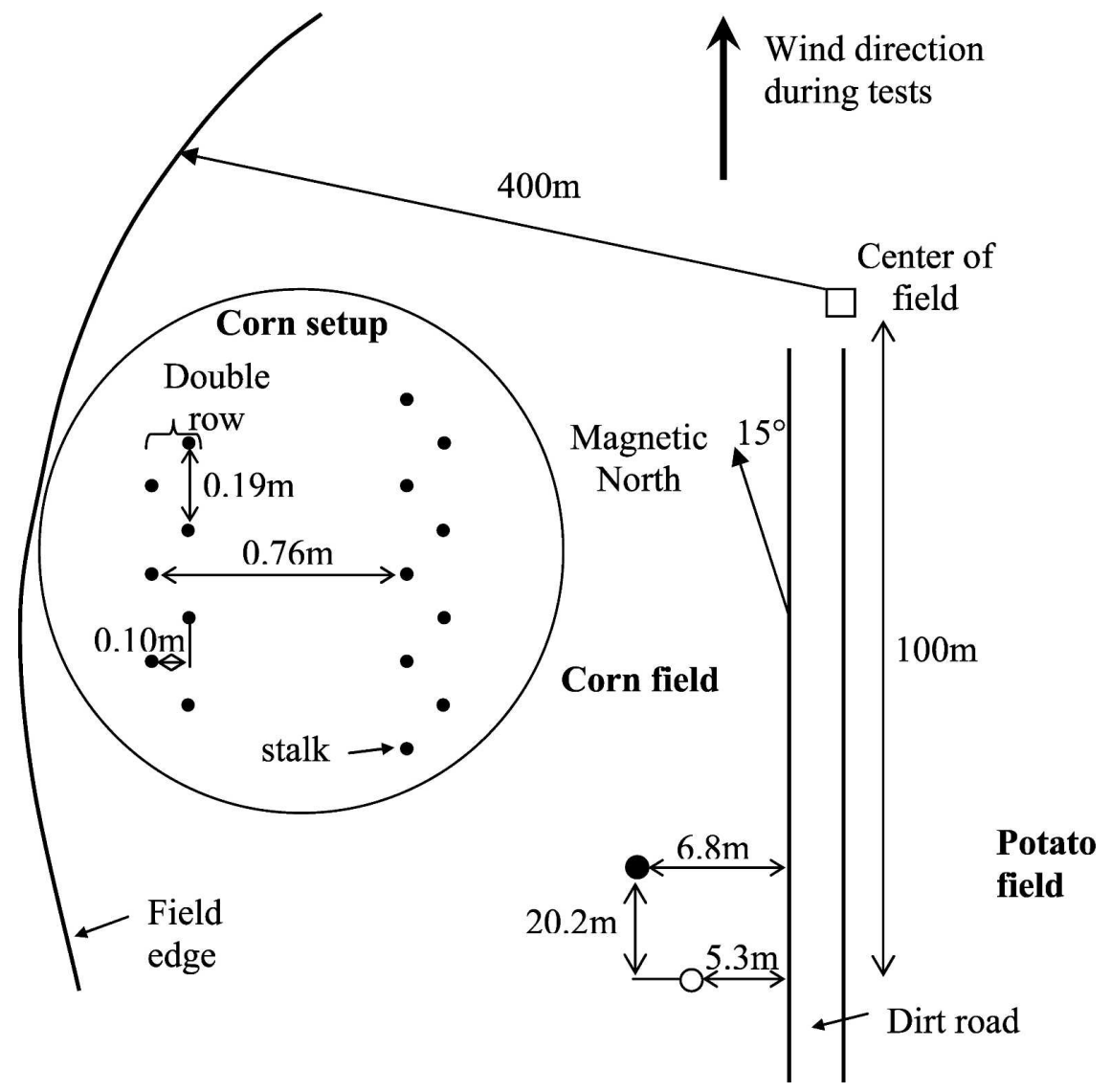

FIG. 1. Schematic layout of the instruments during the field experiment (not to scale): open circle $=$ PIV system; filled circle $=$ meteorological station .

were located about a $100 \mathrm{~m}$ south of the center of the field, and 6.8 and $5.3 \mathrm{~m}$ inside the cornfield, respectively. As discussed later, the wind direction during the tests was southerly, that is, from bottom to top in Fig. 1.

Particle image velocimetry consists of recording two exposures of a section through a flow field seeded with particles that closely follow the flow (Raffel et al. 1998; Adrian 1991). Data analysis provides the instantaneous velocity distribution over the sample area. Thus, the spatial energy spectra can be calculated directly, without invoking Taylor's hypothesis. Data analysis also enables calculation of the spatial distribution of the inplane strain components and the out-of-plane vorticity component.

The field PIV system was essentially the same as the one used by Nimmo Smith et al. (2002), with minor modifications. The laser sheet forming optics and the camera were mounted on a retractable, rotating platform that could be raised up to $9.75 \mathrm{~m}$ above the ground (Fig. 2). A wind vane, mounted on top of the platform, was used for aligning the system with the wind direction.
The light source was a dual flashlamp-pumped dye laser. The laser beam (maximum 120 microjoules per pulse at $594 \mathrm{~nm}$ ) was guided through a $400 \mu$ m-diameter optical fiber to the experimental platform where the beam was expanded to create a vertical light sheet. The thickness of the light sheet varied between 3 and $4 \mathrm{~mm}$ over the sample area. A 12-bit autocorrelation chargecoupled device $(C C D)$ camera $(2048 \times 2048$ pixels, Silicon Mountain Design), having a hardware-based image shifter between exposures to overcome directional ambiguity (Nimmo Smith et al. 2002), was used. The field of view of the camera was $18.2 \times 18.2 \mathrm{~cm}^{2}$, while the distance from the camera to the light sheet was $61.5 \mathrm{~cm}$. The camera was mounted inside a temperaturecontrolled enclosure to prevent overheating.

We used oil-based fog as flow tracers, generated by two Rosco 1600 fog generators. The mean fog particle diameter was $2 \mu \mathrm{m}$ (Han et al. 2002; Kähler et al. 2002). The output nozzle of each generator was connected to a 30-m-long, 10-cm-diameter, perforated, flexible plastic hose, laid out in a half circle of radius $30 \mathrm{~m}$ around the facility inside the cornfield, just below canopy 


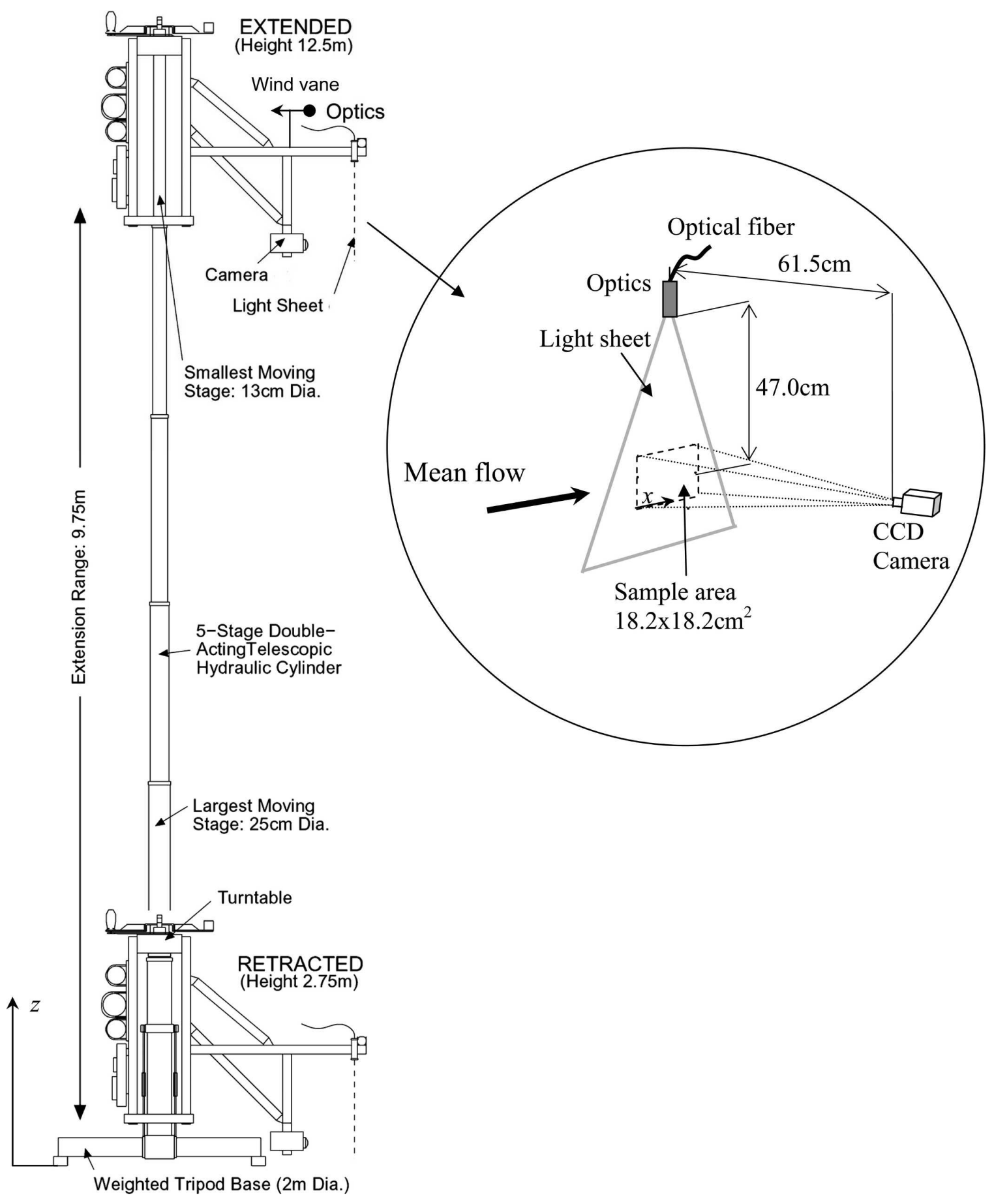

FIG. 2. Schematic layout of the platform carrying the PIV system and the relative position of the camera and the light sheet (not to scale).

height. During the experiments, fog was slowly released through the perforations, and advected toward the experimental facility. For most of the time this approach created uniform seeding in the camera field of view at all elevations.

Out of the 12-bit data we kept and processed the 8 bits that contained the particle traces. The signal-tonoise ratio was subsequently increased using a modified histogram equalization method and then processed using in-house-developed, direct correlation software (Roth and Katz 1999, 2001). We subsequently corrected the data for optical image distortion, and for out-of- 


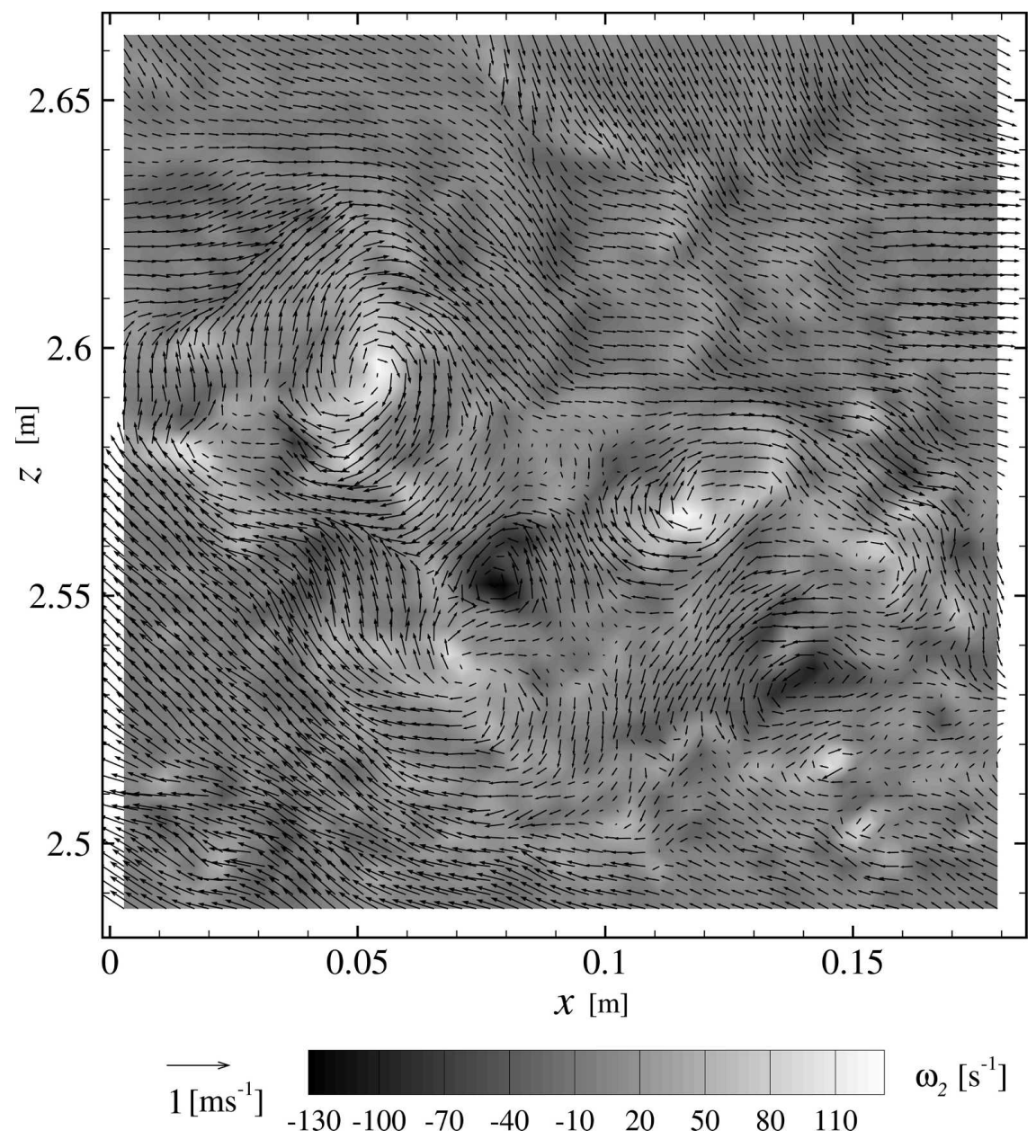

FIG. 3. Typical vector map of the instantaneous velocity field superimposed on the vorticity distribution. The instantaneous spatial mean velocity is subtracted to highlight the flow structure.

plane motion (Nimmo Smith et al. 2002). The interrogation windows were $64 \times 64$ pixels $\left(5.6 \times 5.6 \mathrm{~mm}^{2}\right)$, and with $50 \%$ overlap provided a vector spacing of 2.8 $\mathrm{mm}$. Thus, each instantaneous velocity distribution contained $64 \times 64$ vectors. The uncertainty in the measurements was about 0.2 pixels $\left(0.06 \mathrm{~m} \mathrm{~s}^{-1}\right)$, provided there was sufficient seeding. Since the seeding particles were not always uniformly distributed, we discarded vectors that did not satisfy a minimum correlation coefficient, and subsequently utilized only those maps that contained $70 \%$ or more vectors. The number of vector maps passing our criteria was $3300 \pm 100$. A typical instantaneous velocity field superimposed on the vorticity distribution is shown in Fig. 3. Note that the instantaneous mean velocity (i.e., spatially averaged over the field of view) was subtracted from each vector to highlight the spatial structure of the flow. The resulting $2 \mathrm{D}$ vector maps were used to calculate the ensemble- averaged velocity profiles, turbulence characteristics, vorticity, and spatial spectra. Second-order finite differencing is used for calculating spatial derivatives. In the results that follow, $x_{1}=x, x_{2}=y$, and $x_{3}=z$ are the streamwise, lateral, and wall-normal direction respectively; and $u_{i}\left(u_{1}=u, u_{2}=v, u_{3}=w\right)$ and $u_{i}^{\prime}\left(u_{1}=u^{\prime}\right.$, $\left.u_{2}=v^{\prime}, u_{3}=w^{\prime}\right)$ are the corresponding instantaneous and fluctuating velocity components along $x_{i}$. An overbar is used to indicate ensemble (time) averaging, and a horizontal line average is denoted by angle brackets. For convenience, we denote the ensemble-averaged velocity as $U_{i}(x, z)=\overline{u_{i}}(x, z)$, and $\sigma_{i}$ is the rms value of the corresponding velocity fluctuation component.

The measurements discussed in this paper were carried out during a windy night on 22 July 2003 between 0030 and 0300 eastern daylight time (EDT). The PIV measurements were performed at four different heights: $z / h=1.29,1.20,1.11$, and 0.97 , where $z$ is the 
height of the center of the field of view measured from the ground, and $h=2.67 \mathrm{~m}$ is the average canopy height. At each elevation, 4096 double-exposure images were recorded at an acquisition rate of $4 \mathrm{~Hz}$, corresponding to $1024 \mathrm{~s}$ of sampling time. Before each run the camera was aligned with the wind direction using the wind vane. Based on the meteorological station data (details follow), the 5-min average wind direction did not change by more than $5^{\circ}$ during each run, but standard deviations were $20^{\circ}$. The $3 \mathrm{D}$ sonic anemometers on the meteorological tower were mounted at $z / h=1.0$ and 1.35 , while the RMY was located at $z / h=1.5$. Data from the meteorological station was continuously acquired at $6.9 \mathrm{~Hz}$, using a Campbell Scientific CR23X datalogger, during the entire experiment.

\section{b. Corn field characteristics}

The planted corn variety was 33B51 Pioneer BT gene plus yield guard insecticide. The corn was fully matured and, as illustrated in Fig. 1, it was planted in double rows in a staggered configuration. The lateral distance between rows in a double row was $0.10 \mathrm{~m}$, and the distance between double rows was $0.76 \mathrm{~m}$. The centers of the stalks in each row were longitudinally spaced by $0.19 \mathrm{~m}$. The resulting surface area per plant was $0.1 \mathrm{~m}^{2}$. We measured the height of 43 stalks around the PIV system and obtained an average height of $2.67 \mathrm{~m}$.

The LAI is here defined as the ratio between the total one-sided leaf area and the occupied surface area per plant, while the PFAI is defined as the ratio between the projected frontal area and the occupied surface area of one plant (Finnigan 2000; Wilson et al. 1982). Both were measured using image processing based on four different corn plants located near the measurement site. As illustrated in Fig. 4, for one plant the PFAI was calculated by thresholding the grayscale images, and calculating the projected area of the silhouette shown in Fig. 4b. The LAI was determined in the same way by peeling the leaves as shown in Figs. $4 \mathrm{c}$ and $4 d$. The average results were LAI $=6.0 \pm 0.6$ and $\mathrm{PFAI}=3.7 \pm 0.5$. The ratio $\mathrm{PFAI} / \mathrm{LAI}$ of $0.6 \pm 0.1$ is slightly higher than the typically assumed value in canopy flows (PFAI $=\mathrm{LAI} / 2$; Finnigan 2000). The present LAI is about twice as high as that reported by Wilson et al. (1982), because of the double-row configuration, which halves the ground area per plant.

\section{Experimental results}

\section{a. Mean flow characteristics}

During the tests, the wind was coming from the south-southwest. The mean air temperature, as mea- sured by the Vaisala hygrometer/thermometer, decreased slightly during the experiments, from $26.5^{\circ}$ to $25.8^{\circ} \mathrm{C}$, while the relative humidity increased from $93.5 \%$ to $95.9 \%$. The Obukhov length, $L$ (Kaimal and Finnigan 1994), was calculated using the sonic anemometer data:

$$
L=-\frac{\left(u_{\mathrm{CSAT}}^{*}\right)^{3 / \kappa}}{\left(g / \overline{T_{v}}\right)\left(\overline{u_{3}^{\prime} T_{v}^{\prime}}\right)_{z=h}}
$$

where $\overline{T_{v}}$ is the mean virtual temperature, $\left(\overline{u_{3}^{\prime} T_{v}^{\prime}}\right)_{z=h}$ is the covariance between vertical velocity and virtual temperature fluctuations at canopy height, $g$ is the gravitational acceleration, $\kappa=0.4$ is the von Kármán constant, and the friction velocity is determined from the sonic anemometer located at canopy height, $u_{\text {CSAT }}^{*}=\left[\left(\overline{u_{1}^{\prime} u_{3}^{\prime}}\right)^{2}+\left(\overline{u_{2}^{\prime} u_{3}^{\prime}}\right)^{2}\right]^{1 / 4}$ (Jacobson 1999). The Obukhov length was $20 \mathrm{~m}$, indicating weakly to moderately stable conditions at canopy height.

A comparison between 5-min-averaged streamwise velocities measured by the PIV system (average of the central $10 \times 10$ vectors and 1200 maps), the sonic anemometers (CSAT), and the propeller wind vane (RMY) is shown in Fig. 5. Note that the measurement heights are different, as specified below the graph. An accompanying comparison between the mean flow and turbulence parameters of the PIV and CSAT measurements is presented in Table 1 . The CSAT data are provided only for the cases in which the PIV and CSAT measurements are performed at almost the same height. The PIV data are consistent with the CSATs both in absolute values and trends, considering that measurements are performed at different locations (see Fig. 1). Differences can be attributed to spatial heterogeneity of the flow field, misalignment (less than $10^{\circ}$ ), and differences in elevation between the instruments. The mean wind direction $\left(0^{\circ}\right.$ being a northerly wind) ranges between $190^{\circ}$ and $200^{\circ}$, with standard deviations of each 5-min average of about $20^{\circ}$; that is, it was about parallel to the dirt road, coming from the southsouthwest (Fig. 1). This wind direction falls within $20^{\circ}$ of the orientation of the PIV system. As is evident, the mean velocities range between 1 and $4 \mathrm{~m} \mathrm{~s}^{-1}$, increasing with height.

Normalized mean velocity components and turbulent parameters, nicknamed "family portraits" (Raupach et al. 1996; Finnigan 2000), as calculated from the PIV data, are presented in Fig. 6. The statistics are ensemble averaged over all the datasets at each vector position, and subsequently horizontally line averaged over the 10 central columns. Thus, each plotted point represents an average of more than 30000 measurements. In general, the vertical profiles are consistent with published data 


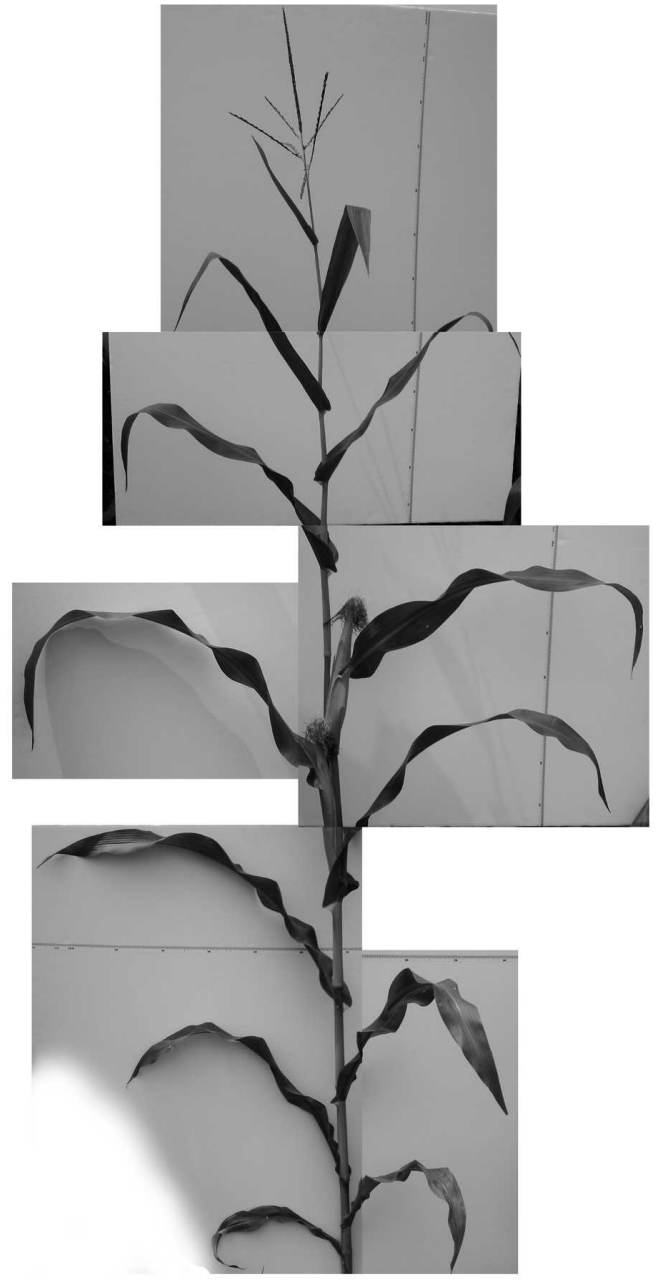

(a)

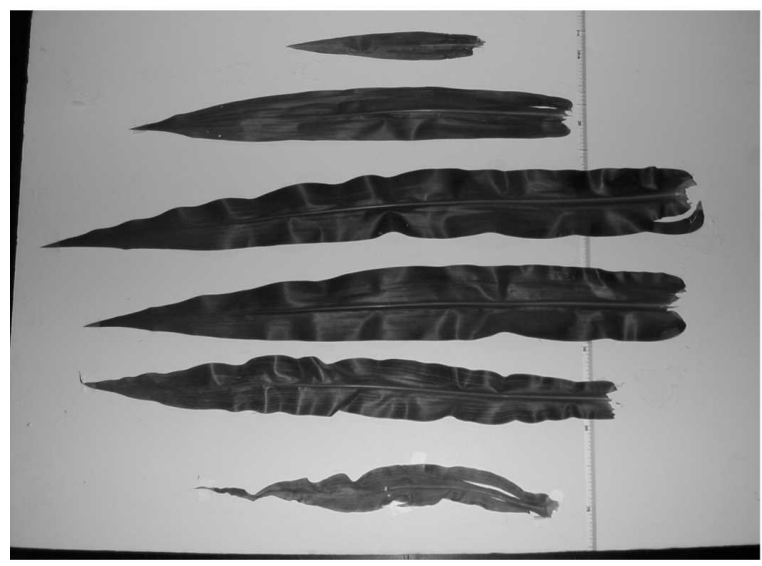

(c)

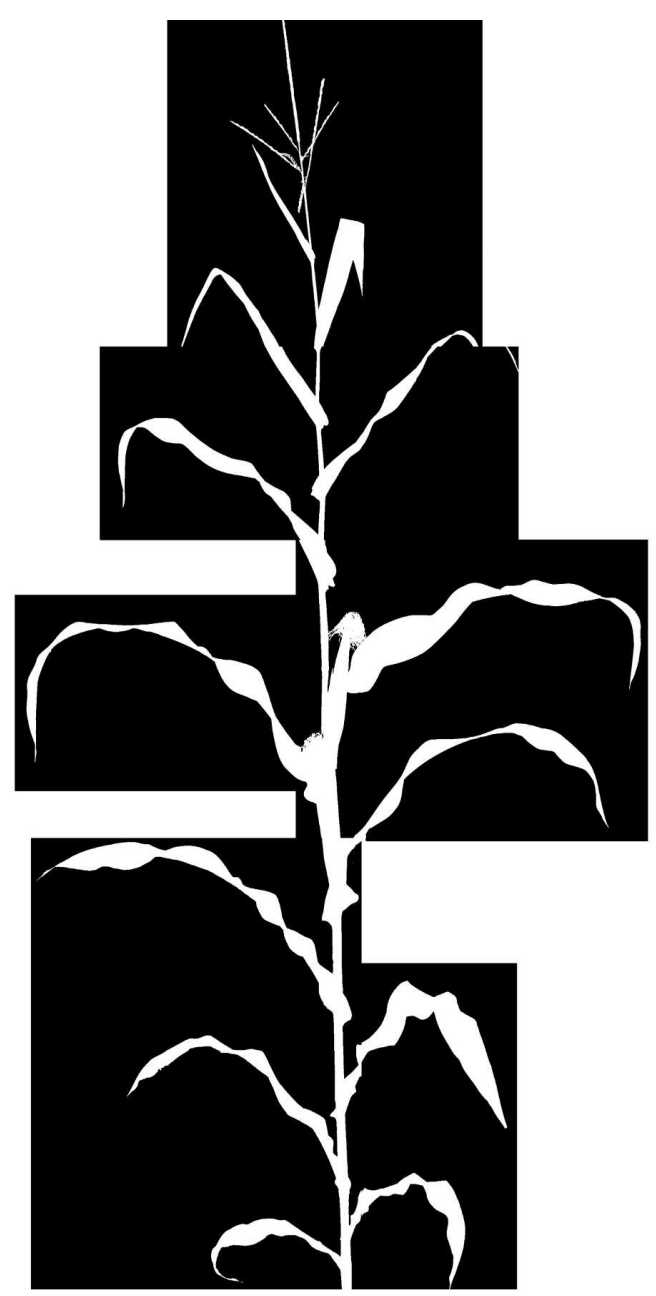

(b)

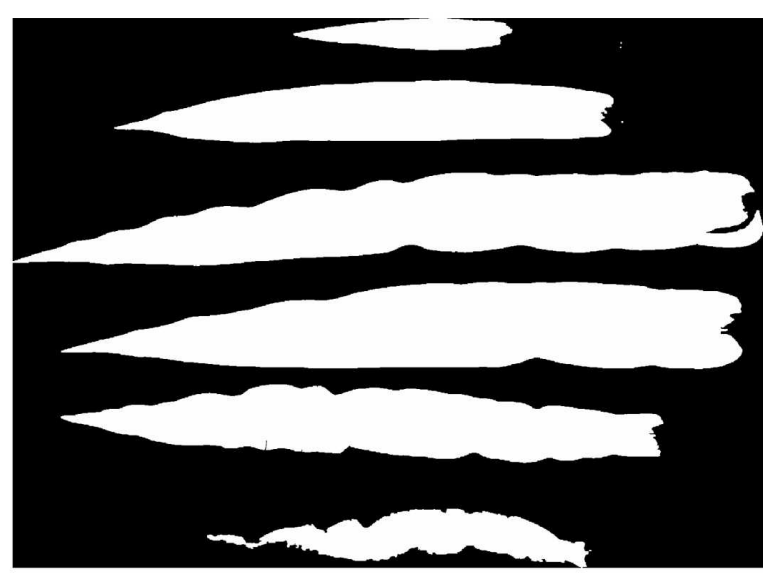

(d)

FIg. 4. Calculation of the LAI and PFAI of corn plants. 


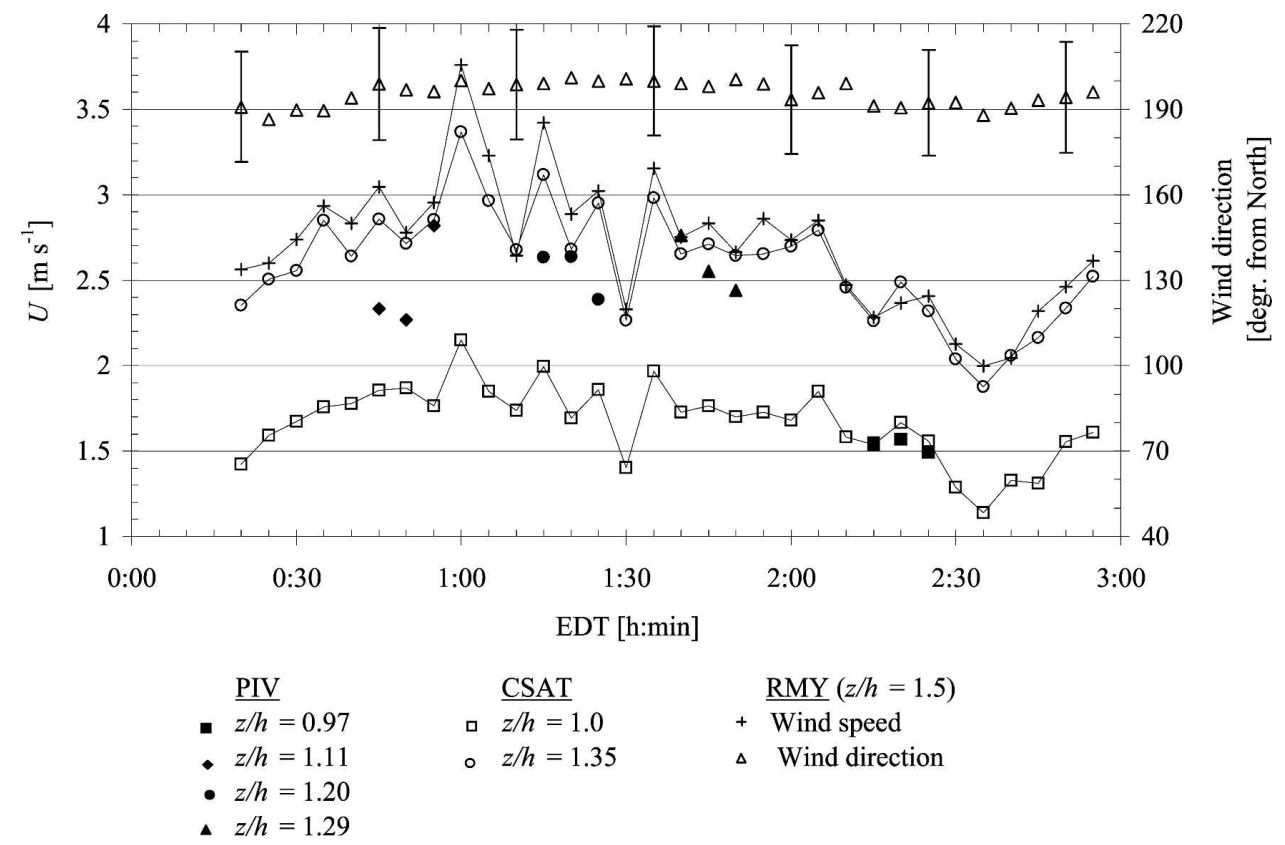

FIG. 5. Five-minute-averaged wind velocities and wind directions on $22 \mathrm{Jul}$ 2003. Error bars indicate standard deviation.

for a variety of canopy flows (Shaw et al. 1974b, 1983; Wilson et al. 1982; Finnigan 2000). In Figs. 6a-c the profiles are normalized by the mean velocity at canopy height, $U_{\mathrm{CSAT}}^{h}$, and the friction velocity, $u_{\mathrm{CSAT}}^{*}$, as measured by the CSAT at the same time (Table 2). Note that the mean velocity changes over time (Fig. 5) and therefore the CSAT mounted at $z / h=1$ is used for normalization since it continuously measures the velocity at canopy height while the PIV system is moved to different elevations. Other parameters presented in Fig. 6 are the correlation coefficient $-\left\langle\overline{u_{1}^{\prime} u_{3}^{\prime}}\right\rangle /\left\langle\sigma_{1}\right\rangle\left\langle\sigma_{3}\right\rangle$, the skewness $\left\langle\overline{u_{i}^{\prime 3}}\right\rangle /\left\langle\sigma_{i}^{3}\right\rangle$, and the locally normalized rms values of velocity fluctuations $\left\langle\sigma_{i}\right\rangle\langle\langle U\rangle$. The normalized shear stress (Fig. 6b) is not equal to unity at canopy height as is usually the case in canopy flows, most likely since the CSAT measurements are performed at a different location than the PIV measurements. In addi- tion, there is the effect of spatial inhomogeneity at the scale of the measurement volume. Figure $6 \mathrm{~b}$ also indicates that the constant shear stress layer is confined to $z / h<1.25$, not like in Finnigan (2000), presumably since the measurements were performed in an advective flow region. In Fig. 6c, as expected, the values of $\sigma_{1}$ are higher than those of $\sigma_{3}$, that is, $\sigma_{1}>\sigma_{3}$. The normalized streamwise velocity fluctuations peak at $1.1<$ $z / h<1.23$, and then decrease at higher elevation. At canopy height, $\sigma_{1}$ and $\sigma_{3}$ are of similar magnitude as those obtained for a wide variety of canopy flows, $\sigma_{1} / u^{*} \approx 2.0$ and $\sigma_{3} / u^{*} \approx 1.0$ (Finnigan 2000). Discrepancies are most likely due to measurements being performed near the edge of the cornfield and in an advective flow region. The correlation coefficient shown in Fig. 6d is approximately 0.5 at canopy height and decreases to about 0.3 at $z / h=1.3$. The former value is

TABLE 1. Comparison of the mean flow and turbulence parameters between CSAT and PIV.

\begin{tabular}{|c|c|c|c|c|c|c|}
\hline \multirow[b]{2}{*}{ Sample time EDT } & PIV & CSAT & \multicolumn{2}{|c|}{ PIV } & PIV & CSAT \\
\hline & \multicolumn{2}{|c|}{$0211-0228$} & 0040-0057 & $0111-0128$ & \multicolumn{2}{|c|}{$0137-0154$} \\
\hline$z / h$ & 0.97 & 1.0 & 1.11 & 1.21 & 1.29 & 1.35 \\
\hline$U_{1}\left(\mathrm{~m} \mathrm{~s}^{-1}\right)$ & 1.49 & 1.54 & 2.50 & 2.46 & 2.60 & 2.67 \\
\hline$U_{3}\left(\mathrm{~m} \mathrm{~s}^{-1}\right)$ & -0.11 & -0.06 & -0.08 & -0.08 & -0.05 & -0.05 \\
\hline$\sigma_{1}\left(\mathrm{~m} \mathrm{~s}^{-1}\right)$ & 0.78 & 0.93 & 1.15 & 1.07 & 0.97 & 1.09 \\
\hline$\sigma_{3}\left(\mathrm{~m} \mathrm{~s}^{-1}\right)$ & 0.47 & 0.44 & 0.66 & 0.66 & 0.64 & 0.59 \\
\hline$-u_{1}^{\prime} u_{3}^{\prime}\left(\mathrm{m}^{2} \mathrm{~s}^{-2}\right)$ & 0.18 & 0.22 & 0.28 & 0.26 & 0.18 & 0.26 \\
\hline
\end{tabular}



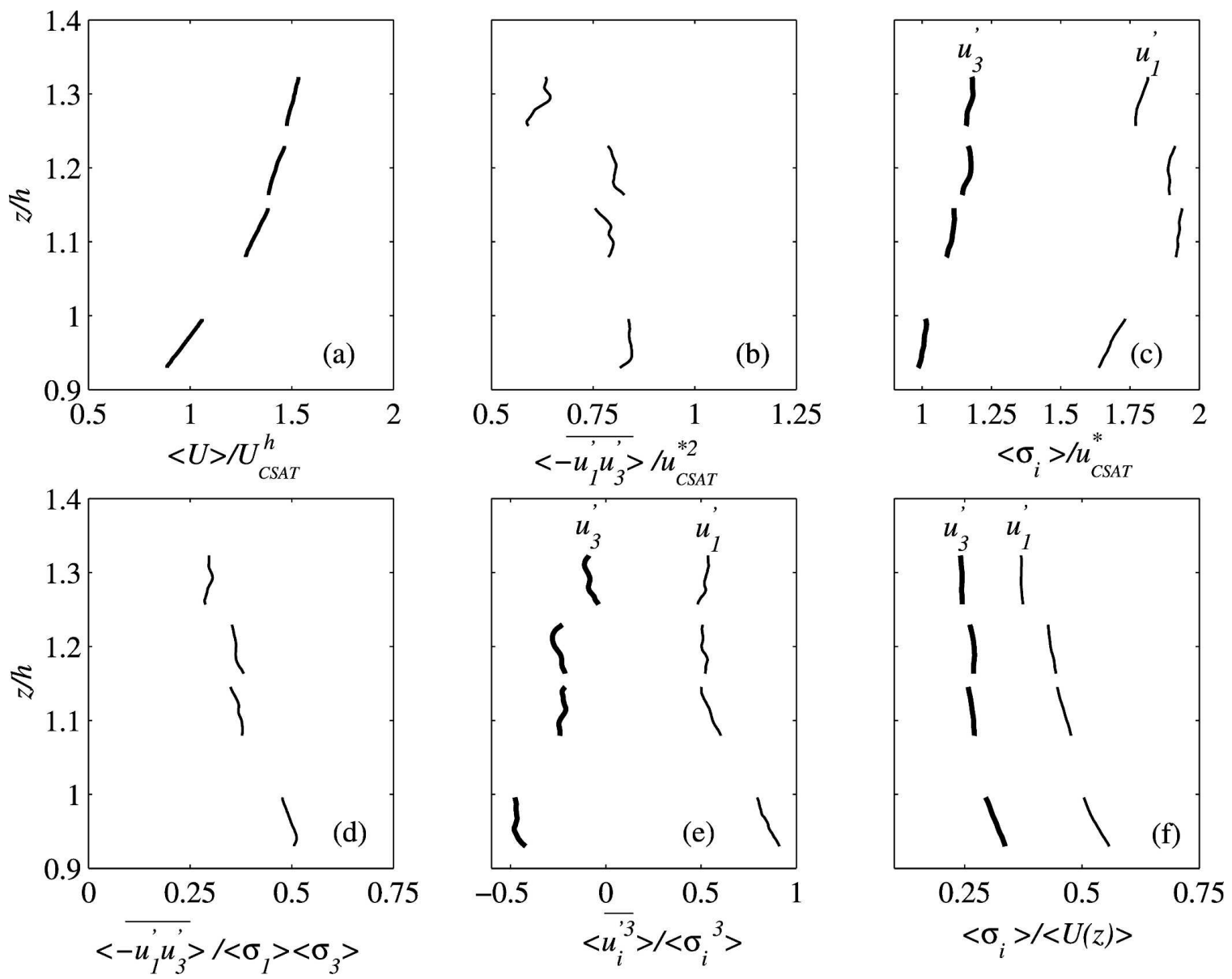

FIG. 6. Mean flow and turbulence statistics of the corn canopy flow.

slightly higher than that occurring in the log layer of a typical turbulent boundary layer, $\sim 0.45$, and the latter is lower (Pope 2000). The elevated level of $-\left\langle u_{1}^{\prime} u_{3}^{\prime}\right\rangle /$ $\left\langle\sigma_{1}\right\rangle\left\langle\sigma_{3}\right\rangle$, which is a measure of the efficiency of vertical momentum transport, does not extend beyond $z / h=$ 1.3. The wall-normal skewness, shown in Fig. 6e, is consistently negative while $\left\langle\overline{u_{1}^{\prime 3}}\right\rangle /\left\langle\sigma_{1}^{3}\right\rangle$ is positive, indicating a preference for sweeping motions $\left(u_{1}^{\prime}>0\right.$ and $\left.u_{3}^{\prime}<0\right)$ near canopy level, as observed before by Shaw et al. (1983). The magnitude of both skewness terms decreases with increasing height. Figure $6 f$ shows that both $x$ and $z$ components of the turbulent intensity increase with decreasing elevation, and that $\left\langle\sigma_{1}\right\rangle /\langle U(z)\rangle$ is larger than $\left\langle\sigma_{3}\right\rangle /\langle U(z)\rangle$. Their magnitudes are similar to those obtained by Shaw et al. (1974b), $\sim 0.4$ and $\sim 0.25$ for the $x$ and $z$ component, respectively.

\section{b. Spectral characteristics and cascading energy flux}

In this section we examine the spectral characteristics of the high-Reynolds-number canopy turbulence. The analysis follows closely the work of Saddoughi and Veeravalli (1994) who measured the spectral characteristics of a turbulent boundary layer in a wind tunnel at slightly lower Taylor scale Reynolds numbers $\left(R_{\lambda} \approx\right.$ $1800)$ compared to the present values $\left(R_{\lambda} \approx 2000-3000\right.$; details follow). In addition we relate our spectra to those obtained in field canopy flows (e.g., Shaw et al. 1974a; Wilson et al. 1982), and in wind tunnel model canopies (e.g., Brunet et al. 1994; Raupach et al. 1986). We also discuss the influence of the interaction be-

TABLE 2. Mean friction velocities and mean velocities at canopy height $(h=2.67 \mathrm{~m})$ used for normalizing the PIV data.

\begin{tabular}{ccc}
\hline \hline \multirow{2}{*}{$\begin{array}{c}\text { Height of PIV } \\
\text { system } z / h\end{array}$} & \multicolumn{2}{c}{ CSAT at $z / h=1.0$} \\
\cline { 2 - 3 } & $u_{\text {CSAT }}^{*}\left(\mathrm{~m} \mathrm{~s}^{-1}\right)$ & $U_{\text {CSAT }}^{h}\left(\mathrm{~m} \mathrm{~s}^{-1}\right)$ \\
\hline .97 & 0.47 & 1.53 \\
1.11 & 0.60 & 1.88 \\
1.20 & 0.57 & 1.74 \\
1.29 & 0.54 & 1.73 \\
\hline
\end{tabular}






FIG. 7. Comparison of $1 \mathrm{D}$ energy spectra obtained from PIV (time series and spatial at $z / h=0.97)$ and CSAT $(z / h=1.0)$.

tween the flow and the canopy elements on the spectral shape and its effect on estimates of dissipation rate.

The spectral characteristics are analyzed using the PIV data in a twofold manner. First, the energy at small scales is examined through spatial spectra that can be calculated directly from the instantaneous velocity distributions. Second, invoking Taylor's "frozen" turbulence hypothesis, the PIV data at each point can be treated as time series $(f=4 \mathrm{~Hz})$ to study the energy at large scales. The latter are compared to the spectra calculated from the sonic anemometer data $(f=6.9$ $\mathrm{Hz}$ ). In all cases we use fast Fourier transforms. To reduce the effect of the finite datasets, we remove the mean and apply linear detrending with zero padding. We do not use a windowing function. Details are provided in Doron et al. (2001) and Nimmo Smith et al. (2005). The spatial spectra presented in this paper are calculated separately for each horizontal line (containing 64 vectors) in the $2 \mathrm{D}$ vector maps, ensemble averaged, and then averaged over the 11 central lines of the velocity distributions. The one-dimensional (1D) spectra $E_{11}, E_{33}$, and $-E_{13}$ are plotted in Fig. 7 as a function of the streamwise wavenumber $\kappa_{1}$. The spectra calculated treating the PIV data as time series, have a range of $4 \times 10^{-3}<\kappa_{1}<8 \mathrm{rad} \mathrm{m}^{-1}$, while the spatial spectra have a range of $34.5<\kappa_{1}<1.1 \times 10^{3} \mathrm{rad} \mathrm{m}^{-1}$. The wavenumbers in the gap between the two plots corre- sponding to length scales ranging from 0.2 to $1 \mathrm{~m}$ are not resolved. The spectra obtained from the PIV and sonic anemometer time series are very similar. The minimal discrepancies can be attributed to the slight misalignments and differences in height. The distributions of $E_{11}$ and $E_{33}$ suggest an inertial range with a $-5 / 3$ slope over almost three decades of wavenumbers. Furthermore, the time series and spatial spectra seem to fit the same $-5 / 3$ line; that is, the spatial spectra appear to be an extension of the time series spectra. Note that the energy in the lowest $\kappa_{1}$ point in each spatial spectrum is reduced because of the effect of detrending. The same agreement persists in the normalized spectra obtained at all heights (Fig. 8). In the inertial range, $E_{33}\left(\kappa_{1}\right) / E_{11}\left(\kappa_{1}\right)$ ranges between 1.41 and 1.48 , that is, slightly above $4 / 3$, the isotropic value, in contrast with Shaw et al. (1974a) who obtained ratios that were smaller than the isotropic value. This difference might be caused by the fact that our spatial spectra only cover the small-scale end of the inertial range, and the start of the dissipation range. As discussed by Finnigan (2000), the shape of the spectra in canopy flows is modified through direct kinetic energy conversion by interaction of both the mean flow and large-scale turbulent structures with canopy elements, into turbulent kinetic energy (TKE) at scales comparable to those of the canopy elements (see also Brunet et al. 1994 and 




FIG. 8. Normalized longitudinal spectra of PIV data at different heights.

Raupach et al. 1986). Consequently, he claims that the spectra deviate from the classical inertial subrange $-5 / 3$ slope including a change in slope and formation of a bump at scales of energy injection. This so-called spectral shortcut mechanism occurs only inside the canopy, in the vicinity of plant elements, but may be "felt" also slightly above canopy because of upward turbulent transport. The measured spectra presented in Figs. 7 and 8 do not show significant deviations from the $-5 / 3$ slope except for the smallest scales, which presumably extend into the dissipation range. Similar $-5 / 3$ slopes were obtained by Shaw et al. (1974a) and Wilson et al. (1982) in a corn canopy. Note that PIV measurements in a wind tunnel laboratory canopy flow model (Zhu et al. 2006) do show significant deviations from a $-5 / 3$ slope below canopy height.

Because of the effect of spectral shortcut, the question arises of how to estimate the cascading energy flux in canopy flows. As we do not resolve the smallest dissipation scales, a direct estimate of dissipation based on the velocity gradients that accounts for all of the dissipation in the sample area is not meaningful. A $-5 / 3$ line fit to the energy spectra, which seems to prevail in our data, does not account for the effect of the bypass mechanism, and will most likely overestimate the cascading energy flux. The modified Kolmogorov theory proposed by Finnigan (2000) is difficult to implement and is only applicable inside the canopy. Another option is to spatially filter the $2 \mathrm{D}$ PIV vector maps and calculate the SGS energy flux as discussed in detail in section 3c. The SGS energy flux directly measures the total energy flux from resolved (filtered) scales to subgrid scales at the filter scale. For isotropic turbulence in the inertial range, the SGS energy flux is almost equal to the dissipation rate (Pope 2000). We show in section $3 \mathrm{c}$ that the SGS energy flux is scale dependent, and that at the smallest scales it is equal to about $40 \%$ of the estimates based on a $-5 / 3$ line fit to the energy spectra. This difference provides a measure of how much the spectrum-based calculation overestimates the cascading energy flux. Thus, in this section, we determine the cascading energy flux by a $-5 / 3$ line fit to the spectra, later assessing its validity by comparing it to the SGS fluxes.

For homogeneous, isotropic turbulence, the 1D longitudinal spectra in the inertial range follow (Pope 2000)

$$
E_{i i}\left(\kappa_{1}\right)=C_{i} \varepsilon^{2 / 3} \kappa_{1}^{-5 / 3},
$$

where $C_{1} \cong(18 / 55) 1.6$ and $C_{3} \cong(24 / 55) 1.6$. Thus by fitting a $-5 / 3$ slope line to the distribution of $E_{i i}$, one can estimate $\varepsilon$, that is, the energy flux cascading down the inertial range. Results are presented in Table 3 along with the SGS flux, $\varepsilon^{\mathrm{SGS}}$.

The Kolmogorov shear stress spectrum decays at a faster rate than the longitudinal spectra. Following Saddoughi and Veeravalli (1994),

$$
E_{13}\left(\kappa_{1}\right)=-C_{13} S \varepsilon^{1 / 3} \kappa_{1}^{-7 / 3}
$$


TABLE 3. Turbulence parameters calculated from the PIV data.

\begin{tabular}{|c|c|c|c|c|c|c|c|c|}
\hline$z / h$ & $\varepsilon\left(\mathrm{m}^{2} \mathrm{~s}^{-3}\right)$ & $\frac{\varepsilon h}{u_{\mathrm{CSAT}}^{* 3}}$ & $\eta(\mu \mathrm{m})$ & $\lambda(\mathrm{mm})$ & $R_{\lambda}\left(10^{3}\right)$ & $\mathcal{L}(\mathrm{m})$ & $\begin{array}{c}\varepsilon^{\mathrm{SGS}} / \varepsilon \\
(\Delta \sim 60 \eta)\end{array}$ & $\frac{3 \nu \overline{\omega_{2}^{2}}}{\varepsilon}$ \\
\hline 0.97 & 0.09 & 2.3 & 440 & 40 & 2.08 & 5.5 & 0.44 & 0.39 \\
\hline 1.11 & 0.18 & 2.2 & 370 & 40 & 3.10 & 8.4 & 0.42 & 0.32 \\
\hline 1.20 & 0.15 & 2.2 & 387 & 41 & 2.92 & 8.6 & 0.40 & 0.32 \\
\hline 1.29 & 0.15 & 2.5 & 387 & 37 & 2.38 & 6.8 & 0.41 & 0.32 \\
\hline
\end{tabular}

where $S$ is the mean shear rate magnitude and $C_{13}$ is a constant. The sample shear spectrum, shown in Fig. 7, is noisier than the normal spectra and seems to decay slightly slower than the $-7 / 3$ slope line, which can be derived from scaling arguments (Pope 2000).

The spectrally estimated dissipation can subsequently be used to calculate the Kolmogorov length scale $\eta=\left(\nu^{3} / \varepsilon\right)^{1 / 4}$, the transverse Taylor microscale $\lambda=$ $\left(15 \nu \sigma_{1}^{2} / \varepsilon\right)^{1 / 2}$, and the Taylor scale Reynolds number $R_{\lambda}=\sigma_{1} \lambda / \nu$, where $\nu$ is the kinematic viscosity of air. The values of $\varepsilon, \eta, \lambda$, and $R_{\lambda}$ are presented in Table 3 for different $z / h$. When the dissipation rates are normalized by $u_{\mathrm{CSAT}}^{* 3} / h$ (also in Table 3 ), their values range between 2.2 and 2.5, consistent with the range of magnitudes obtained in a wind tunnel by Brunet et al. (1994) who show that $\varepsilon h / u_{\mathrm{CSAT}}^{* 3}$ has a broad peak of $\sim 2.5$ at $z / h \approx 1.4$. Within our range of elevations, the highest dissipation rate occurs at $z / h=1.29$. The Kolmogorov length scales range between 370 and $440 \mu \mathrm{m}$, with the highest value obtained at canopy height. The Taylor microscale is almost constant, $\lambda \approx 40 \mathrm{~mm}$, and the corresponding Reynolds numbers range from $R_{\lambda}=$ $2.08 \times 10^{3}$ to $3.10 \times 10^{3}$. Note that $\lambda / \eta$ is of the order of 100 , consistent with ratios obtained by Saddoughi and Veeravalli (1994) and Saddoughi (1997). The integral scale can be calculated using $\mathcal{L}=q^{3 / 2} / \varepsilon$ (Pope 2000). Since we have only data on the in-plane velocity components, the kinetic energy, $q$, is estimated assuming $\overline{u_{2}^{\prime 2}} \cong\left(\overline{u_{1}^{\prime 2}}+\overline{u_{3}^{\prime 2}}\right) / 2$, that is, $q \cong 3\left(\overline{u_{1}^{\prime 2}}+\overline{u_{3}^{\prime 2}}\right) / 4$. The validity of this assumption is supported by the $3 \mathrm{D}$ CSAT measurements. The values of $\mathcal{L}$, also tabulated in Table 3, range between $2 h$ and $3 h$. For $R_{\lambda}>10^{3}$, $\mathcal{L}_{11} / \mathcal{L}=0.43$ (Pope 2000), where $\mathcal{L}_{11}$ is the longitudinal integral length scale. Thus, the present $\mathcal{L}_{11}$ is on the order of $h$, consistent with the literature on canopy flows (e.g., Finnigan 2000).

The normalized longitudinal, 1D spectra at several elevations are plotted in Fig. 8. They have an inertial range extending over three decades, $\sim 10^{-4}<\kappa_{1} \eta<$ $\sim 10^{-1}$. Within the inertial range, all the data collapse onto a single curve, consistent with Saddoughi and Veeravalli (1994). Also consistent is the increase of the inertial range with Reynolds number, although in the present data all the values of $R_{\lambda}$ are of the same order.
The power-law behavior in the inertial subrange is best examined by plotting the compensated Kolmogorov spectrum function, $\Psi(\kappa \eta)$ :

$$
\Psi_{i i}\left(\kappa_{j} \eta\right)=E_{i i}\left(\kappa_{j}\right) \varepsilon^{-2 / 3} \kappa_{j}^{5 / 3} .
$$

Typical examples of $\Psi_{11}\left(\kappa_{1} \eta\right)$ and $\Psi_{33}\left(\kappa_{1} \eta\right)$ are plotted in Fig. 9. The inertial range of the wall-normal component seems to be about one decade shorter than that of the longitudinal component. The compensated spectra tend to reach plateaus in the inertial region whose values are $C_{1}=(18 / 55) 1.6$ and $C_{3}=4 C_{1} / 3$ [Eq. (2)], for the longitudinal and wall-normal component, respectively, suggesting a tendency toward local isotropy. At the low wavenumber end of the inertial range, the wallnormal component is still substantially lower than the isotropic value, consistent with Saddoughi and Veeravalli (1994), while the longitudinal component at canopy height seems to be slightly higher than the isotropic value. At the transition from inertial to dissipation range the wall-normal component seems to exhibit a small hump; that is, small-scale isotropy is not achieved, as discussed in more detail later.

To investigate the power-law behavior of the spatial spectrum in the dissipation region (i.e., for $\kappa_{1} \eta>$ $\sim 10^{-1}$ ), also following Saddoughi and Veeravalli (1994) we plot the compensated spatial spectrum Eq. (4) on log-linear scales in Fig. 10. The dissipation range of the energy spectrum of isotropic turbulence decays as (Kraichnan 1959)

$$
E(\kappa)=A(\kappa \eta)^{\gamma} \exp [-\beta(\kappa \eta)]
$$

The present data for the wall-normal component seem to follow the line plotted for $\beta=5.2$, as obtained previously by direct numerical simulation (e.g., Sanada 1992) and by Saddoughi and Veeravalli (1994). Both spectra are expected to deviate from the $\beta=5.2$ slope because of the effects of noise at high wavenumbers. However, here the streamwise component deviates because of the injection of energy by the spectral shortcut mechanism. Existence of local isotropy in highReynolds-number boundary layer flow was also evalu- 

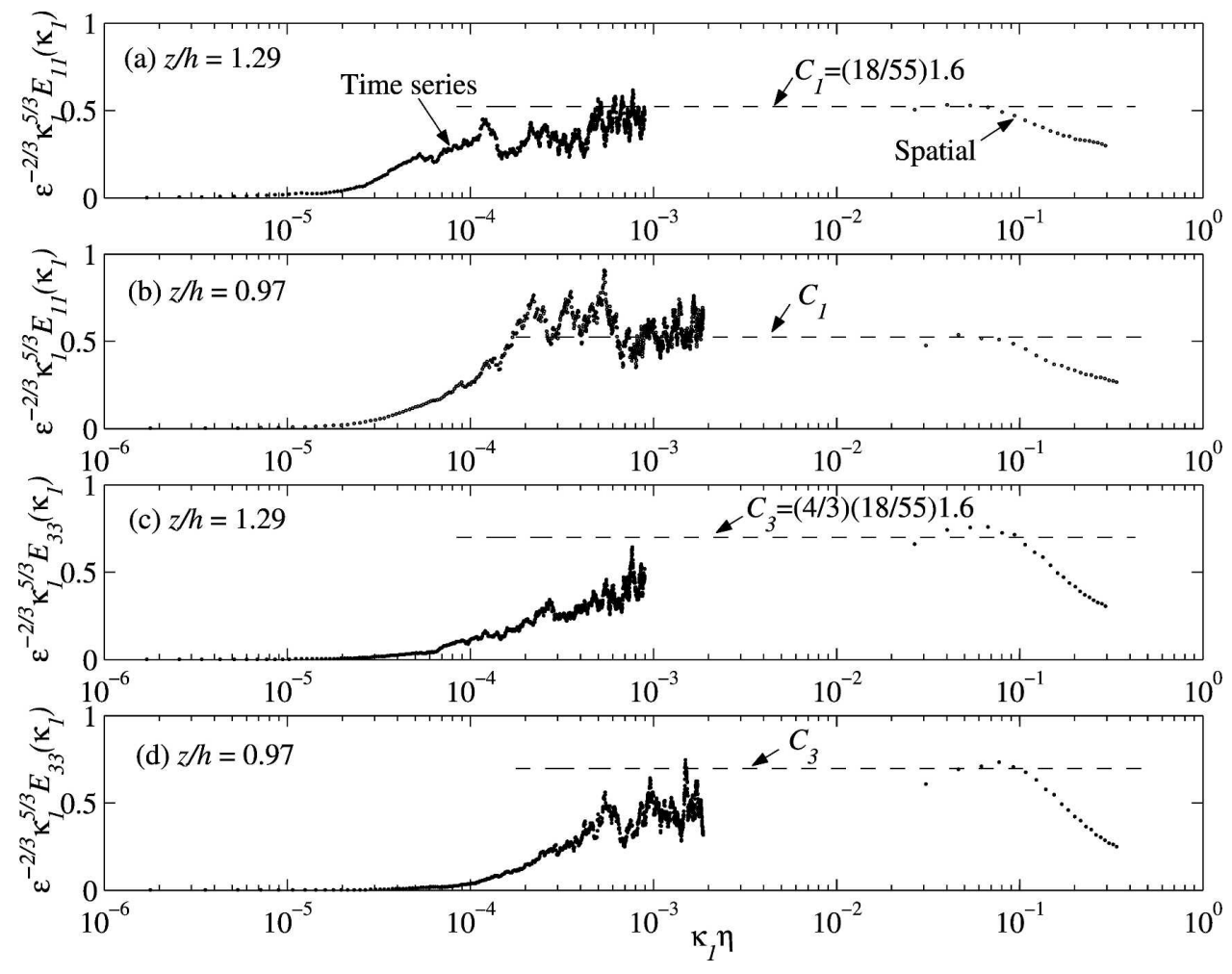

FIG. 9. Compensated longitudinal and wall-normal PIV spectra at $z / h=1.29$ and $z / h=0.97$.

ated by Saddoughi and Veeravalli (1994) using the correlation coefficient:

$$
R_{13}\left(\kappa_{1}\right)=\frac{-E_{13}\left(\kappa_{1}\right)}{\left[E_{11}\left(\kappa_{1}\right) E_{33}\left(\kappa_{1}\right)\right]^{1 / 2}} .
$$

The value of $R_{13}$ should drop to zero as a necessary condition for local isotropy (e.g., Pope 2000). As is evident from Fig. 11, the present values of $R_{13}\left(\kappa_{1}\right)$ indeed decay to zero at $k_{1} \eta \geq 10^{-3}$, with some variations that seem to depend on the local $R_{\lambda}$. However, it is best to keep in mind that symmetries that are less restrictive than isotropy may also lead to the same relation, for example, axial symmetry about the streamwise component (Pope 2000). We do not have out-of-plane data, and as a result, a discussion on what kind of symmetry prevails would be speculative.

Focusing on small scales, we plot in Fig. 12 the dissipation spectra, $\kappa_{1}^{2} E_{11}\left(\kappa_{1}\right)$ and $\kappa_{1}^{2} E_{33}\left(\kappa_{1}\right)$, for the two components, and compare them with the universal spectrum (Gargett et al. 1984; Luznik et al. 2007). The spectral estimates of dissipation are used here without further refinement to match the universal spectra. If values of $\varepsilon$ are adjusted in order to match $\kappa_{1}^{2} E_{11}\left(\kappa_{1}\right)$ (Fig. 12a) with the universal spectrum, the variations of $\varepsilon$ are of the order of $20 \%$. No adjusted values of $\varepsilon$ match $\kappa_{1}^{2} E_{33}\left(\kappa_{1}\right)$ (Fig. 12b) with the universal spectrum. The plots in Fig. 12 are especially sensitive to smallscale anisotropy due to the linear scales involved and since they peak at the wavenumbers of maximum dissipation rate. Clearly, both streamwise and wall-normal spectra deviate from the universal spectra and from each other at $\kappa_{1} \eta>\sim 0.1$, corresponding to a length scale of approximately $2.5 \mathrm{~cm}$, which is of the same order as the corn stem diameter. The streamwise com-

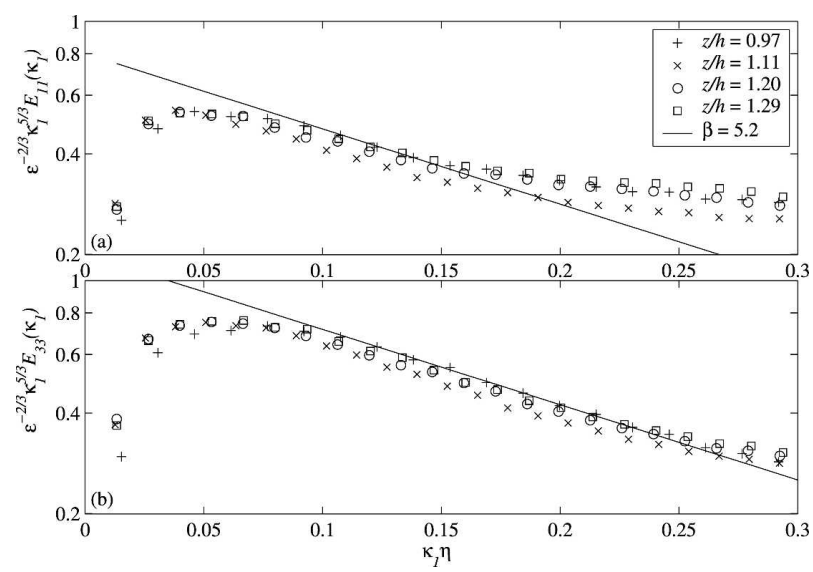

FIG. 10. Compensated semilog plot of the spatial PIV spectra. Solid line is Eq. (5) with $\beta=5$.2. (a) $E_{11}\left(\kappa_{1}\right)$; (b) $E_{33}\left(\kappa_{1}\right)$. 

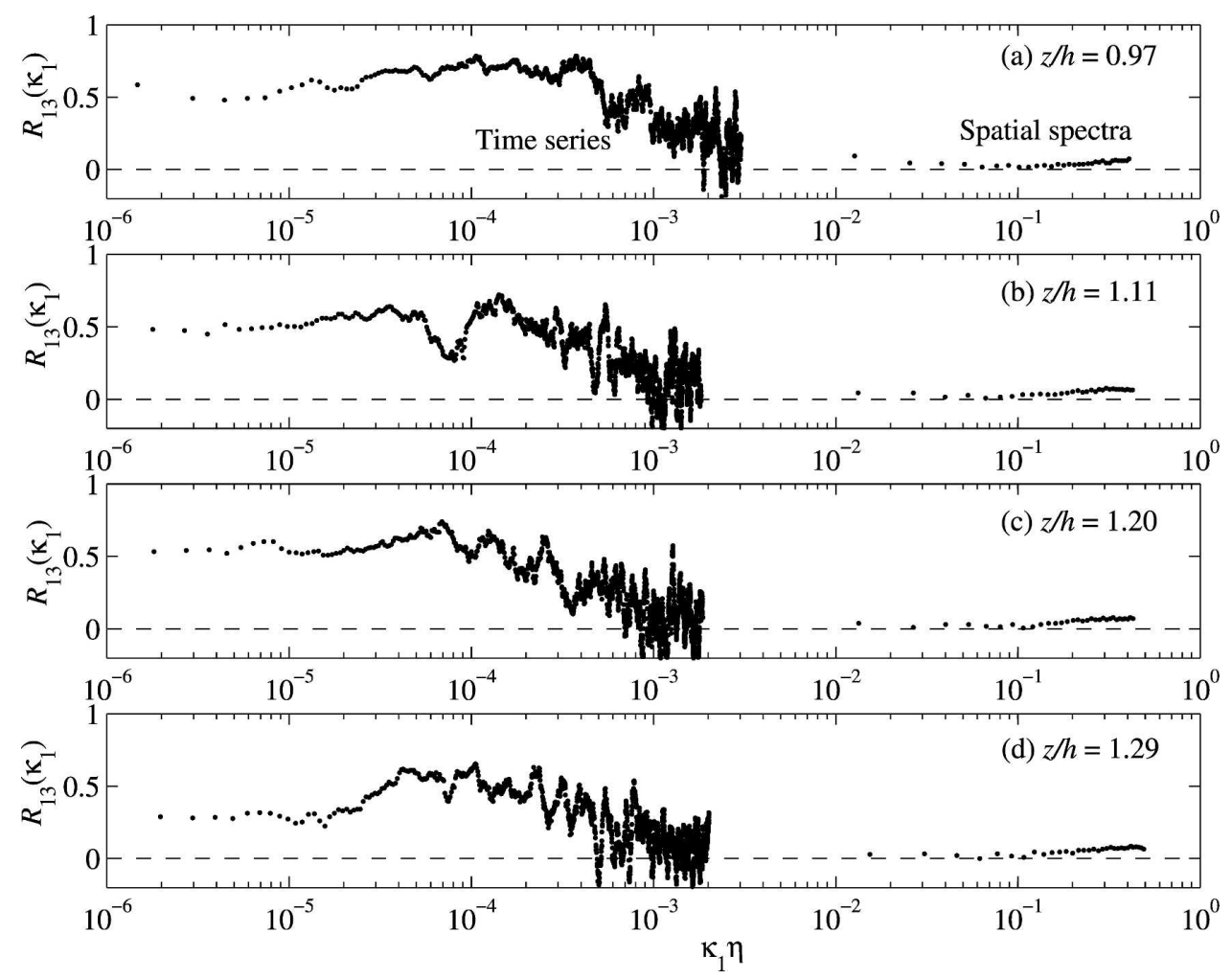

FIG. 11. Spectra of the correlation coefficient $R_{13}\left(\kappa_{1}\right)$ for different heights.

ponent has more energy at small scales than the universal spectrum, whereas as the wall-normal component is less energetic than the universal curve. Although at very high wavenumbers $\left(\kappa_{1} \eta>0.3\right)$ the



spectra are also contaminated by noise, the anisotropy at small scales is evident. These trends might be an indication of injection of energy by the two shortcut mechanisms mentioned before (Finnigan 2000).



FIG. 12. Dissipation spectra plotted with universal dissipation spectra from Gargett et al. (1984). (a) $E_{11}\left(\kappa_{1}\right)$ component, and (b) $E_{33}\left(\kappa_{1}\right)$ component. 


\section{c. Subgrid-scale energy flux}

In this section, we spatially filter the PIV data at different scales, $\Delta$, and calculate the SGS energy flux, $\varepsilon^{\mathrm{SGS}}$, from turbulence at scales larger than $\Delta$, to subgrid scales that are smaller than $\Delta$ (Liu et al. 1994; Chen et al. 2005). This procedure is typically associated with large eddy simulation (LES) of turbulent flow, and the energy flux is the key parameter that SGS stress models attempt to reproduce. In the inertial subrange of homogenous, isotropic turbulence, $\varepsilon^{\mathrm{SGS}}$ is almost equal to $\varepsilon$ (Pope 2000).

The in-plane contribution to the SGS energy flux, $\varepsilon_{2 \mathrm{D}}^{\mathrm{SGS}}(\Delta)$, is (Liu et al. 1999; Chow et al. 2005; Chen et al. 2005)

$$
\varepsilon_{2 \mathrm{D}}^{\mathrm{SGS}}(\Delta)=-\left(\tau_{11} \tilde{S}_{11}+\tau_{33} \tilde{S}_{33}\right)-2 \tau_{13} \tilde{S}_{13}
$$

where $\tau_{i j}={\widetilde{u_{i}}}_{j}-\tilde{u}_{i} \tilde{u}_{j}$ with $i, j=1,3$, is the SGS stress tensor and $\tilde{S}_{i j}=1 / 2\left(\partial \tilde{u}_{i} / \partial x_{j}+\partial \tilde{u}_{j} / \partial x_{i}\right)$ is the resolved (filtered) strain-rate tensor (Meneveau and Katz 2000). The tilde indicates a spatial filtering operation, here a box filter, at some scale $\Delta$. To compare $\varepsilon_{2 \mathrm{D}}^{\mathrm{SGS}}(\Delta)$ to the spectral estimate of dissipation, $\varepsilon$, we use the in-plane contribution to the cascading energy flux in the inertial subrange of homogeneous isotropic turbulence, $\varepsilon_{2 \mathrm{D}}=$ $7 / 15 \varepsilon$ (Fincham et al. 1996). The same relation is applicable to the SGS energy flux, that is, $\varepsilon_{2 \mathrm{D}}^{\mathrm{SGS}}(\Delta)=$ $7 / 15 \varepsilon^{\mathrm{SGS}}(\Delta)$. Figure 13 shows the SGS energy flux normalized by $\varepsilon_{2 \mathrm{D}}$ as a function of filter size $\Delta$. We use filters with sizes of $\Delta=8 \times 8,12 \times 12,16 \times 16$, and $20 \times 20$ vectors, corresponding to approximately 60, 90, 120 , and $150 \eta$. As is evident, the energy transferred from filtered to subgrid scales increases as the filter size decreases. The change is particularly large below canopy height. Above canopy, $\varepsilon_{2 \mathrm{D}}^{\mathrm{SGS}}(\Delta)$ seems to reach a plateau in the $\Delta=60-80 \eta$ range, but not below canopy. Since the increasing energy flux does not cascade down from larger scales, where $\varepsilon_{2 \mathrm{D}}^{\mathrm{SGS}}$ is lower, there must be a different source, consistent with the existence of a spectral bypass mechanism, that injects energy directly to small scales (Finnigan 2000). The size of the corn stem or leaves is about $60-80 \eta$, our smallest filter scale. Unfortunately, we cannot use a significantly smaller filter without adversely affecting the accuracy of the SGS stress calculations. We are also limited by the maximum filter size that still allows us to calculate $\tilde{S}_{i j}$. Interestingly, the values of $\varepsilon_{2 \mathrm{D}}^{\mathrm{SGS}} / \varepsilon_{2 \mathrm{D}}$ range between $28 \%$ and $44 \%$ at all elevations, and are consistent with the ratio between the rate of cascading energy estimated based on Finnigan's (2000) modified Kolmogorov theory, and estimates of dissipation based on $-5 / 3$ line fits to spectra. Therefore, $\varepsilon$ estimated from a $-5 / 3$ line fit overestimates the cascading energy flux but its value is of the

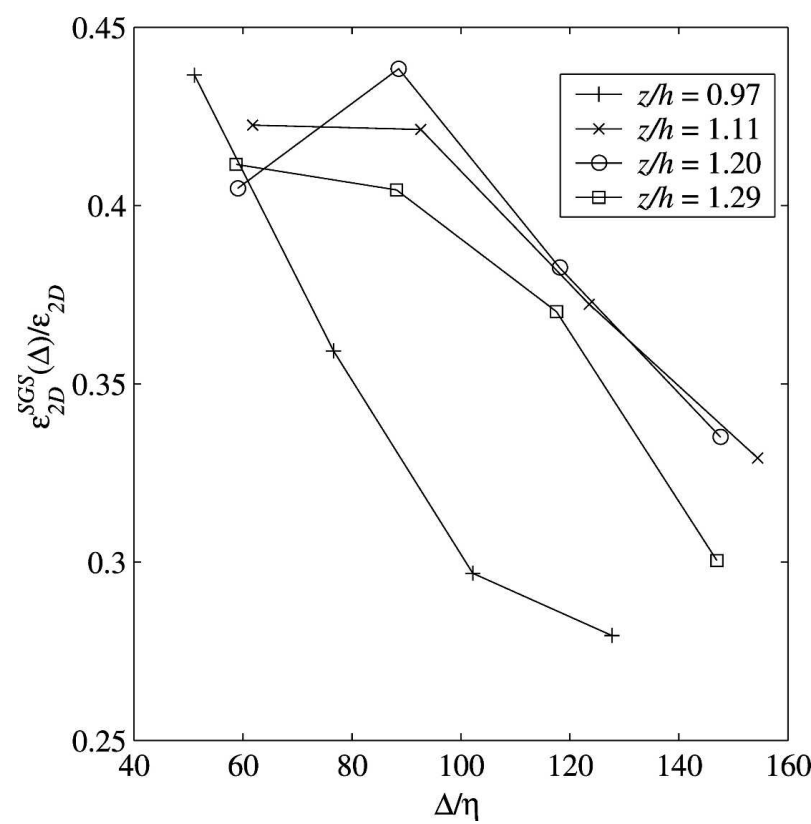

FIG. 13. The ratio of subgrid-scale energy flux to spectrally estimated cascading energy flux.

correct order of magnitude, that is, of the same order as the direct measurement of SGS flux. This conclusion has also been confirmed during wind tunnel measurements within a model canopy (Zhu et al. 2006). For convenience, and since we do not know what filter size to choose and how to account for out-of-plane contributions, we will continue to use the $-5 / 3$ fitted estimates of cascading energy flux in the following sections. However, it is best to keep in mind that the actual cascading energy fluxes are 2.3-3.6 times lower.

\section{d. Terms appearing in the turbulent kinetic energy budget}

TKE budgets have been measured in forest canopies by Leclerc et al. (1990) and Meyers and Baldocchi (1991), and in wind tunnel studies by Raupach et al. (1986) and Brunet et al. (1994). One can use the PIV data to examine several terms in the TKE $\left(\overline{u_{i}^{\prime} u_{i}^{\prime}} / 2\right)$ budget (Pope 2000):

$$
\begin{aligned}
\underbrace{-U_{j} \frac{\partial}{\partial x_{j}}\left(\frac{1}{2} \overline{u_{i}^{\prime} u_{i}^{\prime}}\right)}_{\mathrm{I}}- & \frac{\partial}{\partial x_{j}}(\underbrace{\frac{1}{\rho} \overline{u_{j}^{\prime} p^{\prime}}}_{\mathrm{II}}+\underbrace{\frac{1}{2} \overline{u_{i}^{\prime} u_{i}^{\prime} u_{j}^{\prime}}}_{\mathrm{III}}-\underbrace{2 v \overline{u_{i}^{\prime} s_{i j}}}_{\mathrm{IV}}) \\
& -\underbrace{\overline{u_{i}^{\prime} u_{j}^{\prime} S_{i j}}}_{\mathrm{V}}-\underbrace{2 v \overline{s_{i j} s_{i j}}}_{\mathrm{VI}}=0,
\end{aligned}
$$

where $S_{i j}$ and $s_{i j}$ are the mean and fluctuating rates of strain, respectively, and $p^{\prime}$ is the fluctuating pressure. Here, we do not use the volume-averaged expression of 
TABLE 4. Convective transport of kinetic energy terms (I) normalized by $u_{\mathrm{CSAT}}^{* 3} / h$.

\begin{tabular}{cccc}
\hline \hline$z / h$ & $U_{1} \partial q / \partial x_{1}$ & $U_{3} \partial q / \partial x_{3}$ & $U_{1} \partial q / \partial x_{1+} U_{3} \partial q / \partial x_{3}$ \\
\hline 0.97 & 2.8 & -1.2 & 1.6 \\
1.11 & 4.5 & -0.3 & 4.2 \\
1.20 & 5.6 & -0.2 & 5.4 \\
1.29 & 7.5 & -0.3 & 7.2 \\
\hline
\end{tabular}

the TKE budget since the 3D spatial heterogeneity is not resolved by the present PIV setup. Besides the pressure-velocity correlation term (II), the PIV data should enable us to calculate the in-plane components of all the terms in Eq. (8).

The normalized convective transport of kinetic energy (I), the production rates (V), and turbulent diffusion (III) terms are tabulated in Tables 4,5 , and 6, respectively. For the dissipation rate (term VI) we can only provide estimates, and we do not account for all the dissipation as discussed before. To calculate $U_{j} \partial q /$ $\partial x_{j}$, the gradients are determined by linear least squares fits through the data within the central $20 \times 20$ points of the sample areas. The mean velocities are averaged over the same area. The production rate terms $(\mathrm{V}$, Table 5) are also calculated locally at each point, and then averaged over the central $20 \times 20$ area. Since the light sheet is aligned with the mean flow, the main mean spatial gradients of the mean flow are within the sample area. Thus, we neglect the contributions of $U_{2}$ and $\partial / \partial x_{2}$ terms. In support of this latter assumption, for most of the present data $\partial U_{1} / \partial x_{1}$ is almost equal in magnitude and opposite in sign to $\partial U_{3} / \partial x_{3}$.

We have tried to calculate the gradients of the triple correlation terms (III) based on the spatial distributions in individual sample areas. Unfortunately, even an average of 3300 measurements still leaves significant spatially nonuniform patches, which cause unreasonable variations in gradients, especially when the magnitudes are very small. Thus we do not estimate the streamwise gradients. The only elevation where the streamwise turbulent diffusion appears to provide reasonable values is $z / h=0.97$, where it is extremely small. For the vertical gradients, since we have data at several elevations, that is, a wider range, we can still estimate the terms using a least square fitted power law. The results are presented in Table 6 . The transport by the viscous stresses term (IV) is negligible $\left(<10^{-3}\right)$ at all heights, and as a result it is not presented.

Vertical trends of all terms that we can calculate are summarized in Fig. 14. The terms, including the spectral estimates of dissipation rate, are shown in the same way as in Raupach et al. (1986) and Brunet et al. (1994) to facilitate comparison with previous trends. Here, the loss terms are negative and the gain terms are positive while the dissipation estimate is questionable. Since we do not account for all the terms (e.g., the pressure transport), a balance should not be expected. Indeed, the only elevation where we are close to balance is $z / h=$ 0.97 .

Of the contributors to the production rate (Table 5), $-\overline{u_{3}^{\prime} u_{1}^{\prime}} \partial U_{3} / \partial x_{1}$ is negligible. The terms associated with the mean wall-normal gradients, $-\overline{u_{1}^{\prime} u_{3}^{\prime}} \partial U_{1} / \partial x_{3}$ and

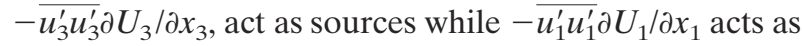
a sink. Although $\partial U_{1} / \partial x_{1}$ is relatively small compared to its vertical gradients, its contribution is amplified by the large $-\overline{u_{1}^{\prime} u_{1}^{\prime}}$. The total production peaks very near to the canopy and decreases at a higher elevation. The production exceeds $\varepsilon$ (at $z / h=0.97)$, but these two "source/sink" terms are comparable above the canopy. The vertical turbulent transport is significant only near the canopy, and decreases with increasing elevation. The trends of production, dissipation rates, and turbulent transport are consistent with the wind tunnel measurements of Brunet et al. (1994) and the field measurements of Leclerc et al. (1990).

\section{$e$. The relation between the dissipation rate and the out-of-plane component of the vorticity}

Boundary layer flows display an intricate pattern of ejection-sweep (E-S) cycles (e.g., Raupach 1981; Shaw et al. 1983; Gao et al. 1989; Finnigan and Shaw 2000) that will be further explored in Part II of this paper (Zhu et al. 2007). A sweep is associated with fastmoving downward-directed fluid, that is, $u_{1}^{\prime}>0$ and $u_{3}^{\prime}<0$, whereas an ejection is associated with slowmoving upward-directed fluid, that is, $u_{1}^{\prime}<0$ and $u_{3}^{\prime}>0$.

TABLE 5. Production terms (V) normalized by $u_{\mathrm{CSAT}}^{* 3} / h$.

\begin{tabular}{cccccc}
\hline \hline$z / h$ & $-\overline{u_{1}^{\prime} u_{1}^{\prime}} \frac{\partial U_{1}}{\partial x_{1}}$ & $-\overline{u_{1}^{\prime} u_{3}^{\prime}} \frac{\partial U_{1}}{\partial x_{3}}$ & $-\overline{u_{3}^{\prime} u_{1}^{\prime}} \frac{\partial U_{3}}{\partial x_{1}}$ & $-\overline{u_{3}^{\prime} u_{3}^{\prime}} \frac{\partial U_{3}}{\partial x_{3}}$ & 1.3 \\
\hline 0.97 & -2.6 & 7.5 & 0.2 & 1.9 & 6.4 \\
1.11 & -2.8 & 4.6 & -0.5 & 2.3 & 3.2 \\
1.20 & -3.8 & 2.9 & -0.3 & 1.1 \\
1.29 & -1.9 & 2.0 & -0.3 & 1.8 \\
\hline
\end{tabular}


TABLE 6 . Turbulent diffusion terms (III) normalized by $u_{\mathrm{CSAT}}^{* 3} / h$.

\begin{tabular}{cccc}
\hline \hline$z / h$ & $\frac{\partial}{\partial x_{3}}\left(\frac{1}{2} \overline{u_{1}^{\prime} u_{1}^{\prime} u_{3}^{\prime}}\right)$ & $\frac{\partial}{\partial x_{3}}\left(\frac{1}{2} \overline{u_{3}^{\prime} u_{3}^{\prime} u_{3}^{\prime}}\right)$ & $\frac{\partial}{\partial x_{3}}\left[\frac{1}{2}\left(\overline{u_{1}^{\prime} u_{1}^{\prime} u_{3}^{\prime}}+\overline{u_{3}^{\prime} u_{3}^{\prime} u_{3}^{\prime}}\right)\right]$ \\
\hline 0.97 & 1.73 & 0.94 & 2.67 \\
1.11 & 0.92 & 0.49 & 1.40 \\
1.20 & 0.66 & 0.35 & 1.02 \\
1.29 & 0.48 & 0.25 & 0.73 \\
\hline
\end{tabular}

As an example, Figs. 15a and $15 \mathrm{~b}$ present the time series of the fluctuating velocity components and their correlation, respectively, at $z / h=1.20$. Time series obtained at different heights appear quite similar and are not shown here. In this analysis, we use the central $20 \times$ 20 vectors of each instantaneous distribution. To highlight trends of large-scale flow features, each data point is an average over $20 \times 20$ instantaneous vectors, and a 30 -s running average. Note that applying a 30 -s running average causes the values of the $-u_{1}^{\prime} u_{3}^{\prime}$ time series to become positive only. Without a running average, $-u_{1}^{\prime} u_{3}^{\prime}$ fluctuates between positive and negative values. These plots provide an insight into the sequence of events occurring in the canopy flow boundary layer. A few ejection-sweep cycles can be clearly discerned in Fig. 15a. As is evident, only about 10 major events occur during the measurement period of $1024 \mathrm{~s}$, raising questions on the statistical significance of the average trends. The characteristic durations of sweep or ejection events are in the 30-50-s range. The time trace of the $-u_{1}^{\prime} u_{3}^{\prime}$ correlation seems to show conflicting trends, at least from visual observations. In some cases, the sweeps involve high correlation, for example, at $300 \mathrm{~s}$, but in other events the shear stresses during sweeps are low, for example, at $70 \mathrm{~s}$. About half of the $-u_{1}^{\prime} u_{3}^{\prime}$ peaks are associated with sweeps. Note, however, that the time trace represents 3D ejection and sweep structures passing the 2D field of view of the camera. While these structures are strong on their centerlines, they become weaker off centerline (Finnigan and Shaw 2000).

The relation between the dissipation rate and the vorticity has been documented in Zhu and Antonia (1997), Nelkin (1999), and Zeff et al. (2003). To the best of our knowledge, it has not been studied in the atmosphere. For isotropic, homogeneous turbulence, the mean dissipation rate is equal to the mean enstrophy, that is, $\varepsilon \equiv \nu \overline{\omega^{2}}$ (e.g., Zhu and Antonia 1997) and $\overline{\omega_{2}^{2}} \equiv$ $\overline{\omega^{2}} / 3$. Unfortunately, our data are too coarse for fully resolving the real vorticity, and/or estimating the dissipation rate from velocity gradients. Furthermore, as discussed before, there is anisotropy at small scales. Still, when we compare the time series of the underresolved out-of-plane enstrophy component, $\omega_{2}^{2}$, to time series of the spectrally estimated, $\varepsilon$, the similarity in trends is striking, as shown in Figs. 15c and 15d. Both time series show running averages over $30 \mathrm{~s}$. To calculate the dissipation time series, we average the spectra of the central 11 rows over $30 \mathrm{~s}$ (1320 spectra) and then fit a $-5 / 3$ line to them. The striking similarity has a correlation coefficient of about 0.9. Furthermore, almost all the major dissipation rate and high enstrophy events occur during sweeps. Conversely, during some of the ejection events, for example, at 900 and $950 \mathrm{~s}$, there are shear stress peaks but the dissipation rate is especially low. However, the magnitudes of $3 v \overline{\omega_{2}^{2}}$ are $2-3$ times smaller than the values of $\varepsilon$, as shown in Table 3 . Insufficient spatial resolution most likely contributes to this discrepancy. Indeed, the values of $3 v \overline{\omega_{2}^{2}}$ are very close to those of the underresolved $7.5 \nu \overline{\left(\partial u_{1}^{\prime} / \partial x_{3}\right)^{2}}$ (not shown), and slightly lower (by 20\%-30\%), than the underresolved $15 \nu \overline{\left(\partial u_{1}^{\prime} / \partial x_{1}\right)^{2}}$ (also not shown). Yet, the values of the underresolved $3 v \overline{\omega_{2}^{2}}$ are comparable to those of $\varepsilon^{\text {SGS }}$ (see Table 3), the energy flux from resolved to subgrid scales. Since the spatial derivatives of velocity components are expected to increase with increasing resolution, the full dissipation (i.e., $2 v \overline{s_{i j} s_{i j}}$ ), would be higher than the present $3 v \omega_{2}^{2}$ (i.e., higher than $\left.\varepsilon^{\mathrm{SGS}}\right)$.

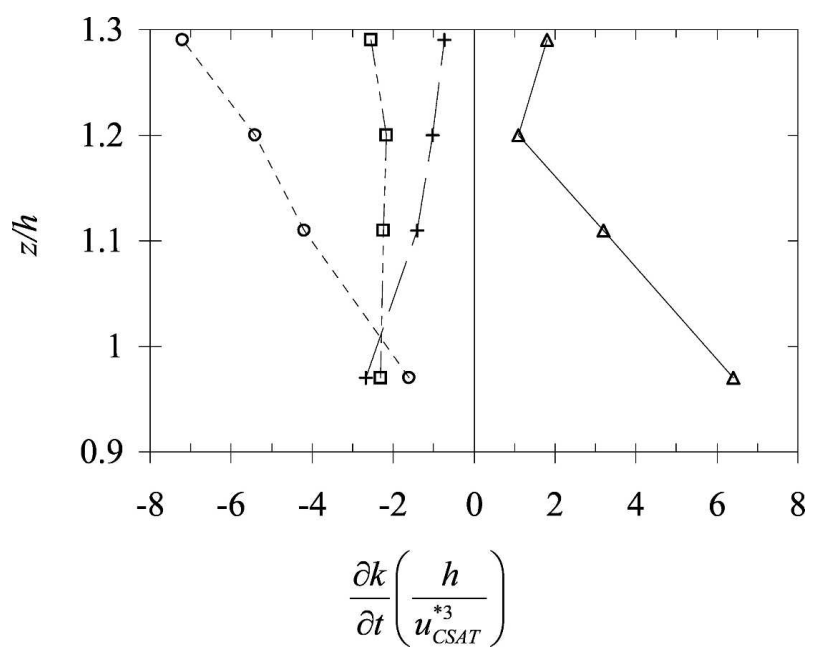

FIG. 14. Normalized terms of the turbulent kinetic energy budget: squares $=\varepsilon($ Table 3$)$; circles $=$ convective terms $($ Table 4$)$; triangles $=$ production $($ Table 5$)$; crosses $=$ vertical turbulent transport (Table 6). 


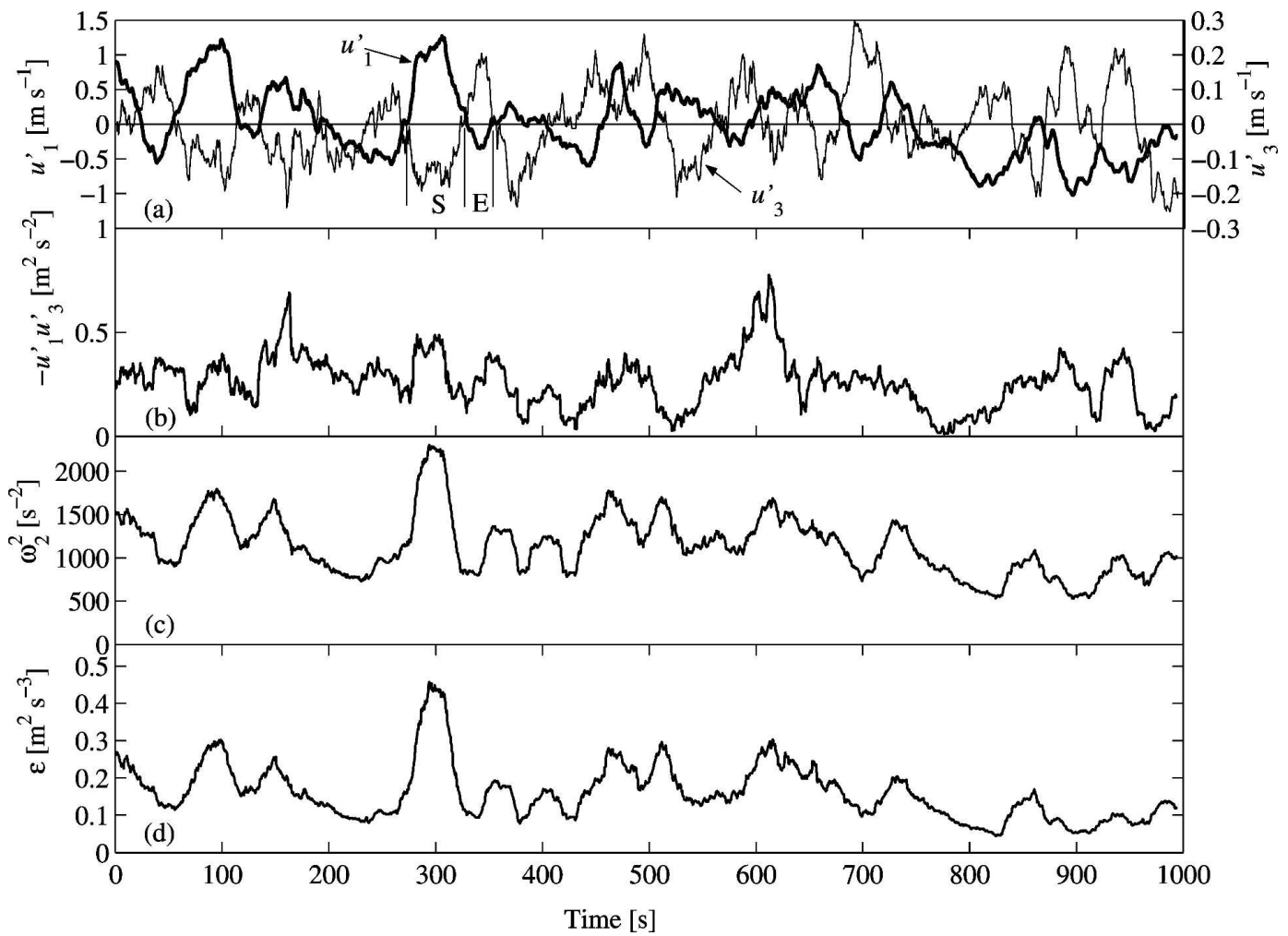

FIG. 15. Time series (30-s running average) at $z / h=1.20$ of (a) $u_{1}^{\prime}, u_{3}^{\prime}$; (b) $-u_{1}^{\prime} u_{3}^{\prime}$; (c) $\omega_{2}^{2}$; and (d) $\varepsilon$.

This strong correlation between vorticity and dissipation has been shown to exist in laboratory flows (Zeff et al. 2003; Zhu and Antonia 1997). Zeff et al. (2003) show that local high strain rates cause vortex stretching, which increases the local growth rate of the vorticity. Thus, the peak in vorticity lags behind the dissipation peak. According to Zeff et al. (2003) the delay is about $0.1-0.2 \tau_{\eta}$. The present delay between samples is about 25 times the Kolmogorov time scale, $\tau_{\eta} \equiv(v / \varepsilon)^{1 / 2} \approx$ $10^{-2} \mathrm{~s}$, and we can therefore not resolve the lag between the enstrophy and dissipation rate peaks.

\section{Summary and conclusions}

PIV measurements along with sonic anemometer and other meteorological sensors examine the flow structure within and above a corn canopy. The mean flow and turbulence statistics measured by PIV compare well with the sonic anemometer data. Furthermore, the trends and absolute values of the mean velocity profiles, rms values of velocity fluctuations, Reynolds stresses, and third-order moments of turbulent velocity fluctuations are consistent with data on canopy flow found in the literature. We construct spectra by combining time and spatial series. The small scales at the transition between inertial and dissipation ranges, from $15 \eta$ to $450 \eta$, are studied using the instantaneous velocity distributions. The low wavenumber range of the spectra is calculated by invoking Taylor's hypothesis on the $4-\mathrm{Hz}$ time series. The spatial spectra appear as a natural extension of the times series spectra in the inertial range. The energy spectra cover five orders of magnitude of length scales; of them the inertial range spans approximately three decades. The shape of the inertial range does not show a noticeable deviation from the classical $-5 / 3$ slope, at least until $\kappa_{1} \eta=0.1$; that is, there is no clear evidence of modifications to the spectral shape due to shortcuts caused by interactions with canopy elements, as suggested by Finnigan (2000). However, note that our data cover the $0.94<z / h<$ 1.32 range; in other words, we may not be deep enough within the canopy to show this effect.

When we examine whether the data satisfy conditions for local isotropy at small scales, for example, by examining the compensated spectra and by calculating the correlation coefficients, at first glance it appears that local isotropy is indeed achieved. However, from plots of the normalized dissipation spectra (which highlight the dissipation scales), it is evident that the trends and magnitudes of vertical and horizontal components 
differ significantly; that is, the flow is clearly not locally isotropic at small scales. Within the dissipation range, especially for $\kappa_{1} \eta \geq 0.1$, the energy of the horizontal component is substantially higher than that of the vertical component. Note that the size of canopy elements is also about $\kappa_{1} \eta \approx 0.1$. Although only accurate to within one order of magnitude, we still estimate the energy flux cascading down in the inertial range by $-5 / 3$ line fits to the energy spectra, and from them obtain that the Kolmogorov length scale is $\eta \approx 0.4 \mathrm{~mm}$, and the Taylor microscale Reynolds numbers range between $R_{\lambda}=2000$ and 3000 . The accuracy of our spectral estimate of the cascading energy flux is evaluated by comparing it to the subgrid-scale (SGS) energy flux, which represents the energy transferred from resolved to subgrid scales in large eddy simulations. The SGS energy flux decreases with increasing filter size; that is, the turbulence at small scales must have an additional energy source that does not cascade down from larger scales. This finding is consistent with the claims that in canopy flows some energy is directly injected into small scales, bypassing the cascading process (Brunet et al. 1994; Finnigan 2000). The SGS flux varies between 0.28 and 0.44 of the value of the cascading energy flux determined by a $-5 / 3$ line fit to the spectra. Thus the spectral values of $\varepsilon$ overestimate the energy flux cascading down the inertial range, but not by orders of magnitude.

The terms appearing in the turbulent kinetic energy budget that can be directly calculated from the 2D PIV data are of the same order and consistent with published data on canopy flows. The total production peaks and exceeds the cascading energy flux at canopy height. With increasing elevation the production rate decreases, while the cascading energy flux remains unchanged. In addition, turbulent transport is significant in the vicinity of canopy height.

Time series of $\varepsilon$ and the out-of-plane component of the vorticity magnitude show a striking similarity, with correlation coefficients of about 0.9 . Time series of velocity fluctuations show a repeated pattern of ejectionsweep cycles, which have a characteristic duration of $30-50$ s. The Reynolds shear stresses show some but not a definite relation to these cycles. Almost all of the high dissipation rate events close to canopy height occur during sweep events. Thus, it is of interest to perform a detailed statistical analysis of these relationships. They are examined in detail in the second part of this paper.

Acknowledgments. The authors thank the anonymous referees that reviewed this manuscript for their insightful review of the original manuscript. Their comments have led to considerable additional analysis and reevaluation of our interpretation of results and conclusions. We thank Mike Embry of the Wye Research and Education Center of the University of Maryland for his help in finding a proper location to conduct the field experiments. We are also grateful to Mac Farms, Inc., in Hurlock, Maryland, for generously granting us access to their cornfield and putting up with our smoke clouds. We are further grateful to Y. Ronzhes and S. King for their technical expertise in developing and maintaining the equipment. Thanks are also due to Prof. G. Brush for support and advice.

This research was funded by the Bio-Complexity Program of the National Science Foundation under Grant 0119903.

\section{REFERENCES}

Adrian, R. J., 1991: Particle-imaging techniques for experimental fluid mechanics. Annu. Rev. Fluid Mech., 23, 261-304.

Amiro, B. D., 1990: Drag coefficients and turbulence spectra within three boreal forest canopies. Bound.-Layer Meteor., 52, 227-246.

Brunet, Y., J. J. Finnigan, and M. R. Raupach, 1994: A wind tunnel study of air flow in waving wheat: Single point velocity statistics. Bound.-Layer Meteor., 70, 95-132.

Chen, J., J. Katz, and C. Meneveau, 2005: Implication of mismatch between stress and strain-rate in turbulence subjected to rapid straining and destraining on dynamic LES models. $J$. Fluids Eng., 127, 840-850.

Chow, Y.-C., O. Uzol, J. Katz, and C. Meneveau, 2005: Decomposition of the spatially filtered and ensemble averaged kinetic energy, the associated fluxes and scaling trends in a rotor wake. Phys. Fluids, 17, doi:10.1063/1.1990206.

Collineau, S., and Y. Brunet, 1993: Detection of turbulent coherent motions in a forest canopy. Part II: Time-scales and conditional averages. Bound.-Layer Meteor., 66, 49-73.

Doron, P., L. Bertuccioli, J. Katz, and T. R. Osborn, 2001: Turbulence characteristics and dissipation estimates in the coastal ocean bottom boundary layer from PIV data. J. Phys. Oceanogr., 31, 2108-2134.

Fincham, A. M., T. Maxworthy, and G. R. Spedding, 1996: Energy dissipation and vortex structure in freely decaying, stratified grid turbulence. Dyn. Atmos. Oceans, 23, 155-169.

Finnigan, J. J., 2000: Turbulence in plant canopies. Annu. Rev. Fluid Mech., 32, 519-571.

— waving wheat: An EOF analysis of the structure of the largeeddy motion. Bound.-Layer Meteor., 96, 211-255.

Gao, W., R. H. Shaw, and K. T. Paw U, 1989: Observation of organized structure in turbulent flow within and above a forest canopy. Bound.-Layer Meteor., 47, 349-377.

Gargett, A. E., T. R. Osborn, and P. W. Nasmyth, 1984: Local isotropy and the decay of turbulence in a stratified fluid. $J$. Fluid Mech., 144, 231-280.

Han, R., G. Papadopoulos, and B. J. Greenspan, 2002: Flow field measurement inside the mouthpiece of the Spiros inhaler using particle image velocimetry. Aerosol Sci. Technol., 36, 329-341.

Jacobson, M. Z., 1999: Fundamentals of Atmospheric Modeling. Cambridge University Press, 656 pp. 
Kähler, C. J., B. Sammler, and J. Kompenhans, 2002: Generation and control of tracer particles for optical flow investigations in air. Exp. Fluids, 33, 736-742.

Kaimal, J. C., and J. J. Finnigan, 1994: Atmospheric Boundary Layer Flows: Their Structure and Measurement. Oxford University Press, $289 \mathrm{pp}$.

Kraichnan, R. H., 1959: The structure of isotropic turbulence at very high Reynolds numbers. J. Fluid Mech., 5, 497-543.

Leclerc, M. Y., K. C. Beissner, R. H. Shaw, G. den Hartog, and H. H. Neumann, 1990: The influence of atmospheric stability on the budgets of the Reynolds stress and turbulent kinetic energy within and above a deciduous forest. J. Appl. Meteor., 29, 916-933.

Liu, S., C. Meneveau, and J. Katz, 1994: On the properties of similarity subgrid-scale models as deduced from measurements in a turbulent jet. J. Fluid Mech., 275, 83-119.

_ subgrid scales during rapid straining of turbulence. J. Fluid Mech., 387, 281-320.

Luznik, L., R. Gurka, W. A. M. Nimmo Smith, W. Zhu, J. Katz, and T. R. Osborn, 2007: Distribution of energy spectra, Reynolds stresses, turbulence production and dissipation in a tidally driven bottom boundary layer. J. Phys. Oceanogr., 37, $1527-1550$.

Meneveau, C., and J. Katz, 2000: Scale-invariance and turbulence models for large-eddy simulation. Annu. Rev. Fluid Mech., 32, $1-32$.

Meyers, T. P., and D. D. Baldocchi, 1991: The budgets of turbulent kinetic energy and Reynolds stress within and above a deciduous forest. Agric. For. Meteor., 53, 207-222.

Nelkin, M., 1999: Enstrophy and dissipation must have the same scaling exponent in the high Reynolds number limit of fluid turbulence. Phys. Fluids, 11, 2202-2204.

Nimmo Smith, W. A. M., P. Atsavapranee, J. Katz, and T. R. Osborn, 2002: PIV measurements in the bottom boundary layer of the coastal ocean. Exp. Fluids, 33, 962-971.

_ - J. Katz, and T. R. Osborn, 2005: On the structure of turbulence in the bottom boundary layer of the coastal ocean. $J$. Phys. Oceanogr., 35, 72-93.

Poggi, D., A. Porporato, L. Ridolfi, J. D. Albertson, and G. G. Katul, 2004: The effect of vegetation density on canopy sublayer turbulence. Bound.-Layer Meteor., 111, 565-587.

Pope, S. B., 2000: Turbulent Flows. Cambridge University Press, $771 \mathrm{pp}$.

Raffel, M., C. E. Willert, and J. Kompenhans, 1998: Particle Image Velocimetry: A Practical Guide. Springer, 253 pp.

Raupach, M. R., 1981: Conditional statistics of Reynolds stress in rough-wall and smooth-wall turbulent boundary layers. $J$. Fluid Mech., 108, 363-382.

—_, and A. S. Thom, 1981: Turbulence in and above plant canopies. Annu. Rev. Fluid Mech., 13, 97-129.

, P. A. Coppin, and B. J. Legg, 1986: Experiments on scalar dispersion within a model plant canopy. Part I: The turbulence structure. Bound.-Layer Meteor., 35, 21-52.

— , R. A. Antonia, and S. Rajagopalan, 1991: Rough-wall turbulent boundary layers. Appl. Mech. Rev., 44, 1-25.

_ J. J. Finnigan, and Y. Brunet, 1996: Coherent eddies and turbulence in vegetation canopies: The mixing layer analogy. Bound.-Layer Meteor., 78, 351-382.

Roth, G. I., and J. Katz, 1999: Parallel truncated multiplication and other methods for improving the speed and accuracy of PIV calculations. Proc. of the Third ASME/JSME Joint Fluids Engineering Conf., San Francisco, CA, ASME, 1-9.

— and - 2001: Five techniques for increasing the speed and accuracy of PIV interrogation. Meas. Sci. Technol., 12, 238245.

Saddoughi, S. G., 1997: Local isotropy in complex turbulent boundary layers at high Reynolds number. J. Fluid Mech., 348, 201-245.

_ , and S. V. Veeravalli, 1994: Local isotropy in turbulent boundary layers at high Reynolds number. J. Fluid Mech., 268, 333-372.

Sanada, T., 1992: Comment on the dissipation-range spectrum in turbulent flows. Phys. Fluids, 4A, 1086-1087.

Shaw, R. H., R. H. Silversides, and G. W. Thurtell, 1974a: Some observations of turbulence and turbulent transport within and above plant canopies. Bound.-Layer Meteor., 5, 429-449.

— C. den Hartog, K. M. King, and G. W. Thurtell, 1974b: Measurements of mean wind flow and three-dimensional turbulence intensity within a mature corn canopy. Agric. Meteor., 13, 419-425.

— , J. Tavangar, and D. P. Ward, 1983: Structure of the Reynolds stress in a canopy layer. J. Climate Appl. Meteor., 22, 1922-1931.

Wilson, J. D., D. P. Ward, G. W. Thurtell, and G. E. Kidd, 1982: Statistics of atmospheric turbulence within and above a corn canopy. Bound.-Layer Meteor., 24, 495-519.

Wolfenbarger, L. L., and P. R. Phifer, 2000: The ecological risks and benefits of genetically engineered plants. Science, 290, 2088-2093.

Zeff, B. W., D. D. Lanterman, R. McAllister, R. Roy, E. J. Kostelich, and D. P. Lathrop, 2003: Measuring intense rotation and dissipation in turbulent flows. Nature, 421, 146-149.

Zhu, W., R. van Hout, L. Luznik, H. S. Kang, J. Katz, and C. Meneveau, 2006: A comparison of PIV measurements of canopy turbulence performed in the field and in a wind tunnel model. Exp. Fluids, 41, 309-318.

- - - $\longrightarrow$, and J. Katz, 2007: PIV measurements in the atmospheric boundary layer within and above a mature corn canopy. Part II: Quadrant-hole analysis. J. Atmos. Sci., 64, 2825-2838.

Zhu, Y., and R. A. Antonia, 1997: On the correlation between enstrophy and energy dissipation rate in a turbulent wake. Appl. Sci. Res., 57, 337-347. 